\title{
A Secure and Efficient Three-Factor Authentication Protocol in Global Mobility Networks
}

\author{
SungJin Yu ${ }^{1}$ (D) Joon Young Lee $^{1}$ (D), YoHan Park ${ }^{2}$, YoungHo Park ${ }^{1, *}$ (i) , SangWoo Lee ${ }^{3}$ \\ and BoHeung Chung ${ }^{3}$ \\ 1 School of Electronics Engineering, Kyungpook National University, Daegu 41566, Korea; \\ darkskiln@knu.ac.kr (S.Y.); harry250@naver.com (J.L.) \\ 2 School of Computer Engineering, Keimyung University, Daegu 42601, Korea; yhpark@kmu.ac.kr \\ 3 Electronics and Telecommunications Research Institute, Daejeon 34129, Korea; ttomlee@etri.re.kr (S.L.); \\ bhjung@etri.re.kr (B.C.) \\ * Correspondence: parkyh@knu.ac.kr; Tel.: +82-53-950-7842
}

Received: 24 March 2020; Accepted: 19 May 2020; Published: 21 May 2020

\begin{abstract}
With the developments in communication and mobile technologies, mobile users can access roaming services by utilizing a mobile device at any time and any place in the global mobility networks. However, these require several security requirements, such as authentication and anonymity, because the information is transmitted over an open channel. Thus, secure and efficient authentication protocols are essential to provide secure roaming services for legitimate users. In 2018, Madhusudhan et al. presented a secure authentication protocol for global mobile networks. However, we demonstrated that their protocol could not prevent potential attacks, including masquerade, session key disclosure, and replay attacks. Thus, we proposed a secure and efficient three-factor authentication protocol to overcome the security weaknesses of Madhusudhan et al.'s scheme. The proposed scheme was demonstrated to prevent various attacks and provided a secure mutual authentication by utilizing biometrics and secret parameters. We evaluated the security of the proposed protocol using informal security analysis and formal security analysis, such as the real-or-random (ROR) model and Burrows-Abadi-Needham (BAN) logic. In addition, we showed that our scheme withstands man-in-the-middle (MITM) and replay attacks utilizing formal security validation automated validation of internet security protocols and applications (AVISPA) simulation. Finally, we compared the performance of our protocol with existing schemes. Consequently, our scheme ensured better security and efficiency features than existing schemes and can be suitable for resource-constrained mobile environments.
\end{abstract}

Keywords: authentication; global mobility networks; roaming service; BAN logic; ROR model; AVISPA simulation

\section{Introduction}

With the advances in wireless communication technology, the global mobility network (GLOMONET) [1-3] has become a popular means of communication. Users can access roaming services through mobile devices; therefore, people's access to knowledge has been improved significantly. In GLOMONET, each mobile user depends on a specific home agent (HA) where they are registered. If the mobile user is in the domain of a foreign agent (FA), the FA must ensure service after authenticating the mobile user. However, as a mobile device has limited resources available in terms of computing power, memory, and battery capacity [4,5], it is not suitable to apply symmetric and asymmetric cryptosystems that generate high computational overheads. In this case, mobile users can face delays during processing and service availing. In addition, a malicious adversary may attempt 
various attacks using sensitive data transmitted via an insecure channel in GLOMONET. Therefore, secure and efficient mutual authentication has become an essential security requirement to provide secure roaming services for legitimate mobile users. The security requirements for GLOMONET are summarized as follows:

- Secure and efficient authentication schemes are required to provide various services in GLOMONET.

- Authentication schemes must resist various attacks, including stolen mobile devices, masquerades, and trace attacks.

- Authentication schemes must consider the limitations of mobile devices relative to the computing power, memory, and battery capacity $[4,5]$.

In the last few years, many authentication schemes have been presented for GLOMONET to ensure the security of users [6-9]. In 2004, Zhu et al. [10] presented an efficient two-factor authentication scheme to provide the roaming facility. However, Lee et al. [11] indicated that Zhu et al.'s [10] protocol did not resist impersonation attacks and also could not achieve user authentication. In 2006, Lee et al. [11] presented an improved protocol for wireless environments to overcome the security flaws of Zhu et al.'s scheme. However, Wu et al. [12] assessed that Lee et al.'s [11] scheme did not withstand perfect backward secrecy and did not ensure user anonymity. In 2012, Li et al. [13] assessed that Wu et al.'s [12] scheme could not withstand replay and masquerade attacks and also could not provide user anonymity.

To overcome these security flaws, Li et al. [13] then proposed a novel user authentication scheme based smart-card to provide efficient high computational and communication overheads. However, Das et al. [14] demonstrated that Li et al.'s protocol [13] was sensitive to replay attacks and did not achieve proper user password updates in the password change processes. In 2015, Marimuthu and Saravanan [15] presented a secure authentication protocol in GLOMONET. However, Madhusudhan et al. [16] proved that their protocol could not withstand offline guessing, insider, stolen-verifier, denial of service, and forgery attacks.

In 2018, Madhusudhan et al. [16] presented a secure and efficient user authentication scheme for GLOMONET using a mobile device to resolve the security problems of Marimuthu and Saravanan's scheme. Madhusudhan et al. claimed that their scheme could prevent replay and masquerade attacks and provide secure mutual authentication. Unfortunately, we analyzed that Madhusudhan et al.'s scheme [16] could not prevent various security threats and could not provide secure mutual authentication. Moreover, Madhusudhan et al.'s scheme [16] was unsuitable for resource-constrained mobile devices as it uses symmetric key encryption and modular multiplication, which generate high computational overheads. Thus, we proposed a secure and efficient three-factor user authentication scheme for roaming services in GLOMONET to resolve the security flaws of Madhusudhan et al.'s scheme.

\subsection{Motivation and Contributions}

We have studied numerous user authentication schemes $[6,8,15,16]$ for roaming services and found that they had the following in common:

1. Many authentication protocols $[6,8,15,16]$ are exposed to well-known attacks, such as masquerade, replay, mobile device theft, and session key disclosure attacks in global mobility environments.

2. Many authentication schemes must provide secure convenience for mobile users in the GLOMONET and must take into account all the security requirements specified in Section 1.2.

3. Secure and lightweight authentication schemes are essential, which take into account limitations for resource-constrained mobile devices relative to computing power, memory, and battery capacity.

Recently, Madhusudhan et al. [16] presented a secure and efficient user authentication scheme for GLOMONET using a mobile device. They claimed that their scheme could resist various attacks 
and could ensure secure mutual authentication and anonymity. However, our paper presents a brief review of Madhusudhan et al.'s scheme [16], and we demonstrated that their scheme could not prevent various security threats. To resolve the security threats of Madhusudhan et al.'s scheme, we proposed a secure and efficient three-factor authentication protocol. The proposed scheme demonstrated several advantages compared with previous related authentication schemes.

First, the proposed scheme could prevent various attacks, such as mobile device theft, masquerade, session key disclosure, and replay attacks and also provided secure mutual authentication, user anonymity, and user friendliness. Second, the proposed scheme used the fuzzy extractor mechanism to improve the security level of the protocol. Even if two of the three factors were compromised, the proposed scheme was still secure. Finally, the proposed scheme provided better effective computation costs with related schemes as it only utilized the one-way hash function. Therefore, the proposed scheme was secure, efficient, and more suitable for practical mobile and wireless environments.

\subsection{Security Requirements}

The research on the security of communication for GLOMONET has shown that the security requirements are essential to produce a secure and efficient authentication protocol. Table 1 shows the security requirements for authenticaiton and key agreement protocol.

Table 1. Security requirements for authentication and key agreement protocols.

\begin{tabular}{ll}
\hline Properties & Description \\
\hline Three-factor security & $\begin{array}{l}\text { This should remain secure even if any two of the three factors } \\
\text { are compromised. }\end{array}$ \\
\hline Resisting known attacks & $\begin{array}{l}\text { This requires that the authentication protocol for GLOMONET is } \\
\text { secure from various known attacks, including privileged insider, } \\
\text { replay, session key disclosure, MITM, and masquerade attacks. }\end{array}$ \\
\hline Resisting stolen mobile device attack & $\begin{array}{l}\text { If an unauthorized person obtains the lost/stolen mobile device, } \\
\text { it is impossible for him to impersonate a valid user with a } \\
\text { counterfeit login request by using the information extracted from } \\
\text { the mobile device. }\end{array}$ \\
\hline Forward and backward secrecy & $\begin{array}{l}\text { This requires that the attacker is not able to obtain the previous } \\
\text { session keys or future ones by using the compromised session key. }\end{array}$ \\
\hline $\begin{array}{l}\text { Secure mutual authentication and } \\
\text { key agreement }\end{array}$ & $\begin{array}{l}\text { This is an essential requirement in the GLOMONET scenario, and } \\
\text { requires the communication parties to be able to authenticate each } \\
\text { other and generate a shared session key to provide confidentiality } \\
\text { of messages in public channels. }\end{array}$ \\
\hline User friendliness & $\begin{array}{l}\text { The mobile user should freely select his/her own identity and } \\
\text { password. In addition, the mobile user should be allowed to } \\
\text { update the password without the assistance of the home agent. }\end{array}$ \\
\hline Anonymity and untraceability & $\begin{array}{l}\text { A malicious attacker is incapable of revealing and tracking the } \\
\text { real identity of the legitimate user, and this is an important } \\
\text { privacy-preserving requirement for users. }\end{array}$ \\
\hline
\end{tabular}

\subsection{Organization}

The remainder of this paper is organized as follows. In Section 2, we present the preliminaries, and in Section 3, we review Madhusudhan et al.'s scheme [16]. In Sections 4 and 5, we assess the security flaws of Madhusudhan et al.'s scheme [16] and present a secure and efficient authentication scheme for GLOMONET to overcome the security flaws of Madhusudhan et al.'s scheme [16]. In Section 6, we demonstrate the security of our scheme using informal security analysis and formal security analysis, including Burrows-Abadi-Needham (BAN) logic and the real-or-random (ROR) model. In Section 7 , 
we report a formal security validation utilizing the Automated Validation of Internet Security Protocols and Applications (AVISPA) simulation tool. In Section 8, we compare the performance properties of our protocol to existing protocols. We present our conclusions in the final Section 9.

\section{Preliminaries}

This section presents preliminaries to facilitate reader comprehension.

\subsection{Attacker Model}

To examine the security of our protocol, we describe the Dolev-Yao (DY) model [17], which is described as follows:

- An adversary is able to eavesdrop, intercept, modify, delete, or insert messages exchanged through an open channel.

- An adversary is able to obtain the lost or stolen mobile device of legitimate mobile users $[18,19]$ and can extract the important data stored in the mobile device by utilizing a power-analysis attack $[20,21]$.

- An adversary is able to perform various types of attacks, including replay, masquerade, man-in-the-middle (MITM) and mobile device theft attacks.

\subsection{Fuzzy Extractors}

This section discusses the basic concepts of a fuzzy extractor. According to [22], this mechanism involves two procedures, such as Gen and Rep. The detailed description for Gen and Rep are below:

1. Gen: After a user imprints the biometric input Bio, the probabilistic function Gen selects a consistent random string $\rho \in\{0,1\}^{l}$ and a random auxiliary string $\sigma \in\{0,1\}^{*}$.

2. Rep : After a new user imprints the biometric input Bionew and the string value $\sigma$ in a session, Rep successfully recovers the value $\rho$.

\section{Review of Madhusudhan et al.'s Protocol}

Madhusudhan et al.'s scheme [16] is comprised of three processes: (1) user registration, (2) authentication, and (3) password update. The notations utilized in this paper are defined in Table 2 and each process is detailed as follows.

Table 2. Notations.

\begin{tabular}{ll}
\hline Notation & Description \\
\hline$I D_{M U}$ & $M U^{\prime}$ s identity \\
\hline$I D_{F A}$ & $F A^{\prime}$ s identity \\
\hline$I D_{H A}$ & $H A^{\prime}$ 's identity \\
\hline$R_{S}$ & $H A^{\prime}$ s random number \\
\hline$R_{M U}, R_{F A}, R_{H A}$ & Random nonce of $M U, F A$, and $H A$ \\
\hline$P W_{M U}$ & $M U^{\prime}$ s password \\
\hline$B I O_{i}$ & $M U^{\prime}$ s biometrics \\
\hline$K_{S}$ & $H A^{\prime}$ s master key \\
\hline$S K_{i}$ & Session key between $M U$ and $F A$ \\
\hline$K_{F H}$ & Shared secret key between $F A$ and $H A$ \\
\hline$(X)_{K}$ & Symmetric encryption/decryption \\
\hline$T$ & Timestamp \\
\hline$h(\cdot)$ & Hash function \\
\hline$\oplus$ & Bitwise XOR operation \\
\hline$\|$ & Concatenation operation \\
\hline
\end{tabular}




\subsection{Initialization Process}

The home agent $(H A)$ selects two prime numbers $p, q$ and generator $g$ of a finite field in $Z_{p}^{*}$, of which $Z_{p}^{*}$ is a nonsingular elliptic curve $y^{2}=x^{3}+a x+b(\bmod p)$. The $H A$ calculates $n=p \times q$ and $\phi(n)=(p-1) \times(q-1)$. Then, the $H A$ chooses an integer $e$, such that $1<e<\phi(n)$ and $\operatorname{gcd}(e, \phi(n))=1$. After that, the $H A$ computes the value of an integer $d$, such that $d=e^{-1}$, where $d$ is the secret key of the $H A$, and $y=g^{d} \bmod n$, where $y$ is the public key of the $H A$. The $H A$ keeps $\{p, q, d\}$ securely.

\subsection{Registration Process}

In Madhusudhan et al.'s protocol, a new $M U$ who requests roaming services must register their identity with the HA. Figure 1 indicates the user registration process of Madhusudhan et al.'s protocol [16] and this process is described in detail as follows.

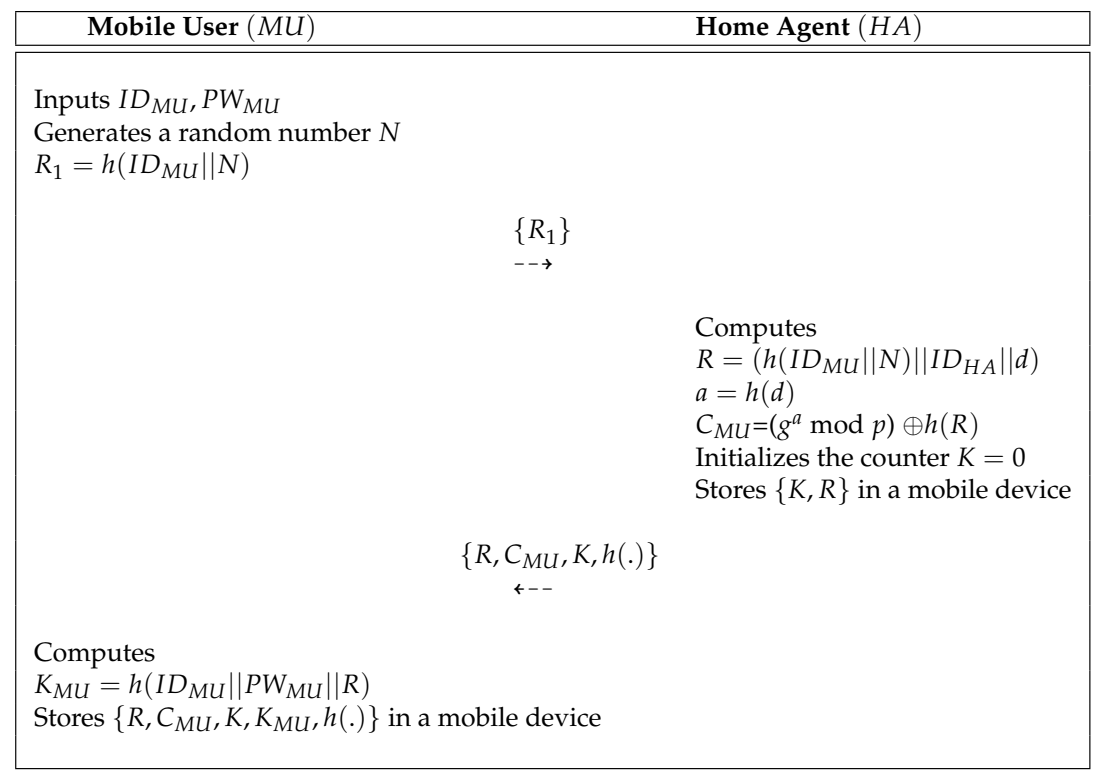

Figure 1. The user registration process of Madhusudhan et al.'s protocol.

Step 1: A mobile user $M U$ inputs $I D_{M U} a n d P W_{M U}$ and selects a random number $N$. Then, $M U$ computes $R_{1}=h\left(I D_{M U} \| N\right)$ and sends a request message to the $H A$ via a public channel.

Step 2: After obtaining messages $\left\{R_{1}\right\}$, the $H A$ calculates $R=\left(h\left(I D_{M U} \| N\right)\left\|I D_{H A}\right\| d\right), a=h(d)$ and $C_{M U}=\left(g^{a} \bmod p\right) \oplus h(R)$. After that, $H A$ sets the value of the counter $K=0$ and stores $\{K, R\}$ in a secure database. Then, $H A$ sends $\left\{R, C_{M U}, K, h().\right\}$ to $M U$ over a secure channel.

Step 3: After obtaining messages $\left\{R, C_{M U}, K, h().\right\}$, the $M U$ computes $K_{M U}=h\left(I D_{M U}\left\|P W_{M U}\right\| R\right)$ and stores it in a mobile device. Finally, the mobile device of the $M U$ contains $\left\{R, C_{M U}, K, K_{M U}, h().\right\}$.

\subsection{Login and Authentication Process}

In Madhusudhan et al.'s protocol [16], they considered a scenario in which the $M U$ associated with the $H A$ visits a foreign network from the foreign agent $F A$ and attempts to access the roaming service. A $M U$ who requests roaming service must send a login request message to the $H A$. The $M U$, $F A$, and $H A$ then perform mutual authentication with each other, then $M U$ and $F A$ share the session key. Figure 2 indicates the login and authentication process of Madhusudhan et al.'s protocol [16]. The process is described in detail as follows. 


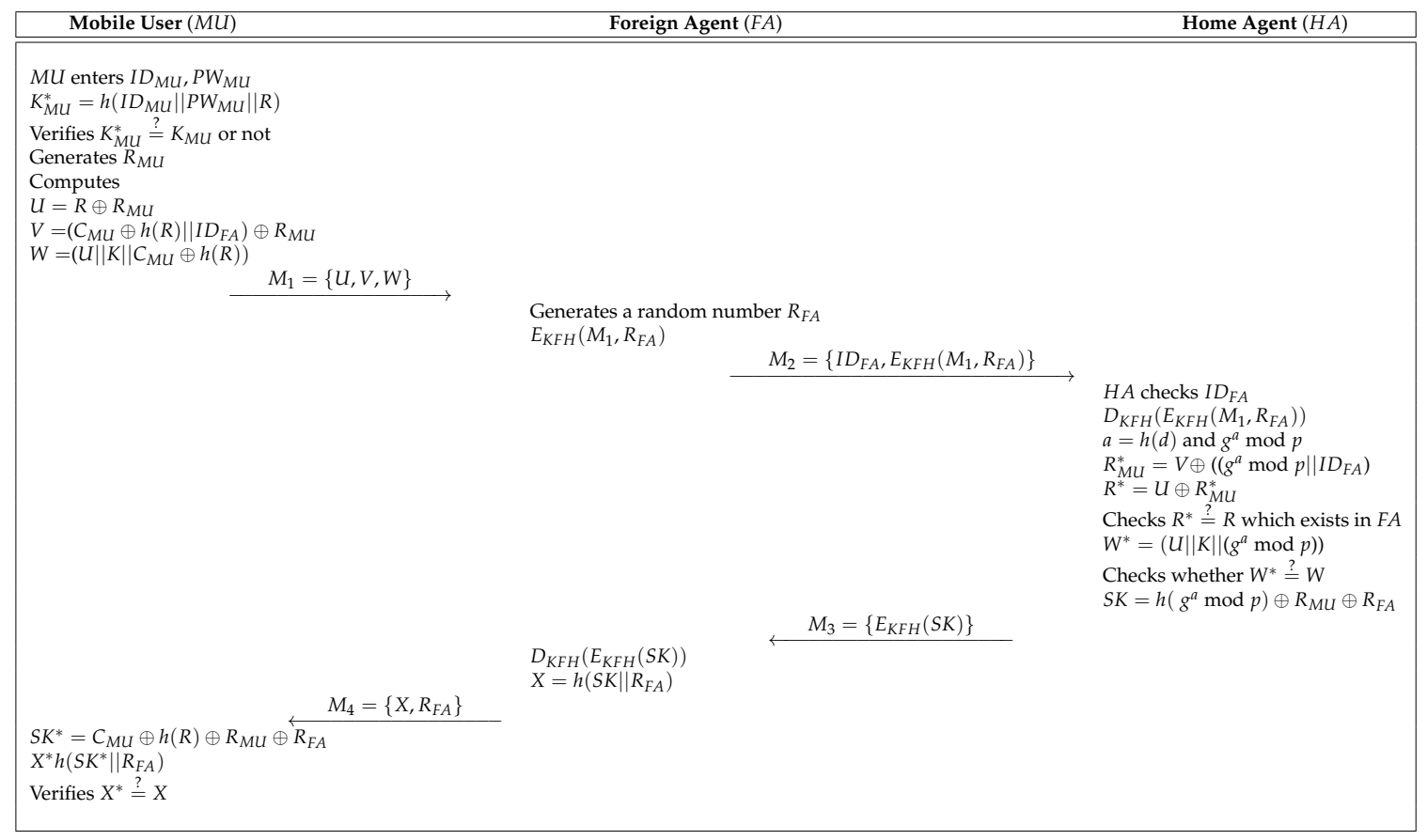

Figure 2. The login and authentication process of Madhusudhan et al.'s protocol.

Step 1: The $M U$ retrieves the authentication data stored in the mobile device and enters $I D_{M U}$ and $P W_{M U}$. After that, the mobile device computes $K_{M U}^{*}=h\left(I D_{M U}\left\|P W_{M U}\right\| R\right)$ and checks whether $K_{M U}^{*} \stackrel{?}{=} K_{M U}$. If this condition holds, the $M U$ generates a random number $R_{M U}$ and computes $U=R \oplus R_{M U}, V=\left(C_{M U} \oplus h(R) \| I D_{F A}\right) \oplus R_{M U}$ and $W=\left(U\|K\| C_{M U} \oplus\right.$ $h(R))$. Then, $M U$ sends the login request message $M_{1}=\{U, V, W\}$ to $F A$ through an insecure channel.

Step 2: After obtaining the $M_{1}=\{U, V, W\}, F A$ selects a random number $R_{F A}$ and encrypts $M_{1}$ and $R_{F A}$ using the shared secret key. The $F A$ sends the $M_{2}=\left\{I D_{F A}, E_{K F H}\left(M_{1}, R_{F A}\right)\right\}$ to the HA.

Step 3: Upon reception of $M_{2}=\left\{I D_{F A}, E_{K F H}\left(M_{1}, R_{F A}\right)\right\}$, the $H A$ checks the identity $I D_{F A}$ of $F A$ and retrieves the secret key corresponding to $I D_{F A}$. After that, the $H A$ decrypts $D_{K F H}\left(E_{K F H}\left(M_{1}, R_{F A}\right)\right)$ and computes $a=h(d), g^{a} \bmod p, R_{M U}^{*}=V \oplus\left(\left(g^{a} \bmod p\right) \| I D_{F A}\right)$ and $R^{*}=U \oplus R_{M U}^{*}$. The $H A$ then checks whether there exists $R^{*} \stackrel{?}{=} R$ in a secure database. If the condition is valid, the $H A$ computes $W^{*}=\left(U\|K\|\left(g^{a} \bmod p\right)\right)$ and checks whether $W^{*} \stackrel{?}{=} W$. If it is correct, the $H A$ computes $S K=h\left(g^{a} \bmod p\right) \oplus R_{M U} \oplus R_{F A}$ and sends $M_{3}=\left\{E_{K F H}(S K)\right\}$ to the $F A$.

Step 4: After obtaining the $M_{3}=\left\{E_{K F H}(S K)\right\}$, the $F A$ decrypts $D_{K F H}\left(E_{K F H}(S K)\right)$ and computes $X=h\left(S K \| R_{F A}\right)$. Finally, the $F A$ sends $M_{4}=\left\{X, R_{F A}\right\}$ to the $M U$.

Step 5: Upon reception of $M_{4}=\left\{X, R_{F A}\right\}$, the $M U$ computes $S K^{*}=C_{M U} \oplus h(R) \oplus R_{M U} \oplus R_{F A}$ and $X^{*}=h\left(S K^{*} \| R_{F A}\right)$. After that, the $M U$ checks whether $X^{*} \stackrel{?}{=} X$. If this holds, the $M U$ and $F A$ achieve the $S K$ successfully.

\subsection{Password Update Process}

In Madhusudhan et al.'s protocol, the $M U$ can freely update their password. The process is described in detail as follows.

Step 1: When a legitimate $M U$ wants to update the password, the $M U$ inputs $I D_{M U}, P W_{M U}$ and the request messages are transmitted via a terminal. 
Step 2: $\quad$ The mobile device of $M U$ calculates $K_{M U}^{*}=h\left(I D_{M U}|| P W_{M U}\right)$ and checks whether $K_{M U}^{*} \stackrel{?}{=}$ $K_{M U}$. If this holds, the $M U$ is legitimate user. Otherwise, the mobile device terminates the password change process.

Step 3: The $M U$ selects new password $P W_{M U}^{N E W}$ and computes $K_{M U}^{N E W}=h\left(I D_{M U} \| P W_{M U}^{N E W}\right)$. Finally, the mobile device of $M U$ replaces $\left\{K_{M U}\right\}$ with $\left\{K_{M U}^{N E W}\right\}$.

\section{Cryptanalysis of Madhusudhan et al.'s Protocol}

We demonstrated the security shortcomings of the existing protocol [16]. They claimed that their scheme can resist replay and masquerade attacks and achieve secure user authentication. However, we demonstrated that Madhusudhan et al.'s protocol [16] is insecure against various attacks, including session key disclosure, replay, and masquerade attacks. Furthermore, we show that the existing protocol [16] does not provide mutual authentication.

\subsection{Masquerade Attack}

If a malicious adversary $M U_{a}$ can attempt to impersonate a legitimate user, $M U_{a}$ can easily generate the message $M_{1}=\{U, V, W\}$ of the legitimate user. As discussed in Section 2.1, $M U_{a}$ obtains the mobile device of $M U$ and extracts the stored secret parameters in it. In addition, $M U_{a}$ intercepts the message exchanged over a public channel. Finally, $M U_{a}$ performs the masquerade attack and its detailed procedures.

Step 1: $\quad$ A $M U_{a}$ calculates $R_{M U}=U \oplus R, V=\left(C_{M U} \oplus h(R) \| I D_{F A}\right) \oplus R_{M U}$ and $W=\left(U\|K\| C_{M U} \oplus\right.$ $h(R))$. Then, $M U_{a}$ generates a random number $R_{a}$. After that, $M U_{a}$ computes $U_{a}=R \oplus$ $R_{a}, V_{a}=\left(C_{M U} \oplus h(R) \| I D_{F A}\right) \oplus R_{a}$ and $W_{a}=\left(U_{a}\|K\| C_{M U} \oplus h(R)\right)$ and sends $M_{1 a}=$ $\left\{U_{a}, V_{a}, W_{a}\right\}$ to the $F A$.

Step 2: After obtaining the $M_{1 a}=\left\{U_{a}, V_{a}, W_{a}\right\}$, the $F A$ selects a random number $R_{F A}$ and encrypts $E_{K F H}\left(M_{1}, R_{F A}\right)$ using a shared secret key. Then, the $F A$ sends $M_{2}=\left\{I D_{F A}, E_{K F H}\left(M_{1 a}, R_{F A}\right)\right\}$ to the $H A$.

Step 3: Upon reception of $M_{2}=\left\{I D_{F A}, E_{K F H}\left(M_{1 a}, R_{F A}\right)\right\}$, the $H A$ decrypts $D_{K F H}\left(E_{K F H}\left(M_{1}, R_{F A}\right)\right)$ and computes $a=h(d), g^{a} \bmod p, R_{a}^{*}=V_{a} \oplus\left(\left(g^{a} \bmod p \| I D_{F A}\right)\right.$ and $R^{*}=U_{a} \oplus R_{a}^{*}$. Then, the $H A$ checks whether $R^{*} \stackrel{?}{=} R$. After that, $H A$ computes $W_{a}^{*}=\left(U_{a}\|K\|\left(g^{a} \bmod p\right)\right)$ and checks whether $W^{*} \stackrel{?}{=} W$. Finally, $H A$ computes $S K=h\left(g^{a} \bmod p\right) \oplus R_{a} \oplus R_{F A}$ and sends $M_{3}=\left\{E_{K F H}(S K)\right\}$ to the $F A$.

Step 4: After obtaining the $M_{3}=\left\{E_{K F H}(S K)\right\}$, the $F A$ decrypts $D_{K F H}\left(E_{K F H}(S K)\right)$ and computes $X=h\left(S K \| R_{F A}\right)$, then sends $M_{4}=\left\{X, R_{F A}\right\}$ to the $M U_{a}$.

Step 5: Upon reception of $M_{4}=\left\{X, R_{F A}\right\}$, the $M U_{a}$ computes the $S K^{*}=C_{M U} \oplus h(R) \oplus R_{a} \oplus R_{F A}$, $X^{*}=h\left(S K^{*} \| R_{F A}\right)$ and checks whether $X^{*} \stackrel{?}{=} X$. If it is correct, $M U_{a}$ computes the $S K$

$M U_{a}$ obtains the session key between $M U_{a}$ and $F A$ and performs mutual authentication successfully. As a result, Madhusudhan et al.'s protocol [16] is insecure against the masquerade attacks.

\subsection{Replay Attack}

Madhusudhan et al. claimed that their protocol can withstand replay attacks because a $M U_{a}$ cannot calculate the correct $S K=h\left(g^{a} \bmod p\right) \oplus R_{M U} \oplus R_{F A}$ without the random number $R_{F A}$ and $R_{M U}$. However, according to Section 4.1, $M U_{a}$ computes $R_{M U}=U \oplus R$ and obtains $R_{F A}$ in an open channel. Furthermore, $M U_{a}$ can extract the secret parameter $\left\{C_{M U}, R\right\}$ stored in the mobile device. $M U_{a}$ computes $S K=C_{M U} \oplus h(R) \oplus R_{M U} \oplus R_{F A}$. In addition, according to Section 2.1, $M U_{a}$ can obtain the counter value $K$ in the mobile device. Thus, Madhusudhan et al.'s protocol [16] is insecure against replay attacks. 


\subsection{Session Key Disclosure Attack}

According to Section 4.1, a $M U_{a}$ can successfully impersonate a legitimate mobile user $M U$ and calculate the $S K$. According to the discussion presented in Section 2.1, $M U_{a}$ can extract the $\left\{C_{M U}, R\right\}$ in the mobile device and obtain random number $R_{F A}$ of $F A$ over an open channel, and then compute $R_{M U}=U \oplus R$. Therefore, $M U_{a}$ can compute $S K=C_{M U} \oplus h(R) \oplus R_{M U} \oplus R_{F A}$. Therefore, Madhusudhan et al.'s protocol [16] is insecure against session key disclosure attacks.

\subsection{Mutual Authentication}

In the existing protocol [16], they indicated that their scheme preserves secure mutual authentication among the $M U, F A$, and $H A$. However, according to Section 4.1, their protocol cannot prevent masquerade attacks and the $M U_{a}$ can successfully calculate authentication request message $W=\left(U|| K|| C_{M U} \oplus h(R)\right)$ and authentication message $X^{*}=h\left(S K^{*} \| R_{F A}\right)$. Consequently, Madhusudhan et al.'s protocol [16] cannot achieve mutual authentication.

\section{Proposed Secure and Efficient Authentication Protocol for GLOMONET}

Many biometric-based user authentication protocols $[23,24]$ have been presented to improve the security flaws associated with mobile device authentication. Biometric-based schemes are difficult to guess, duplicate, and forge and cannot be stolen or lost. Therefore, biometric-based three-factor authentication mechanisms are more secure than mobile device and password based two-factor authentication mechanisms. Therefore, we present a secure and efficient authentication protocol using biometrics to overcome the security problems of the existing protocol [16].

\subsection{Registration Process}

A new $M U$ should register with $H A$ to receive the roaming services. Figure 3 presents the user registration process of our protocol.

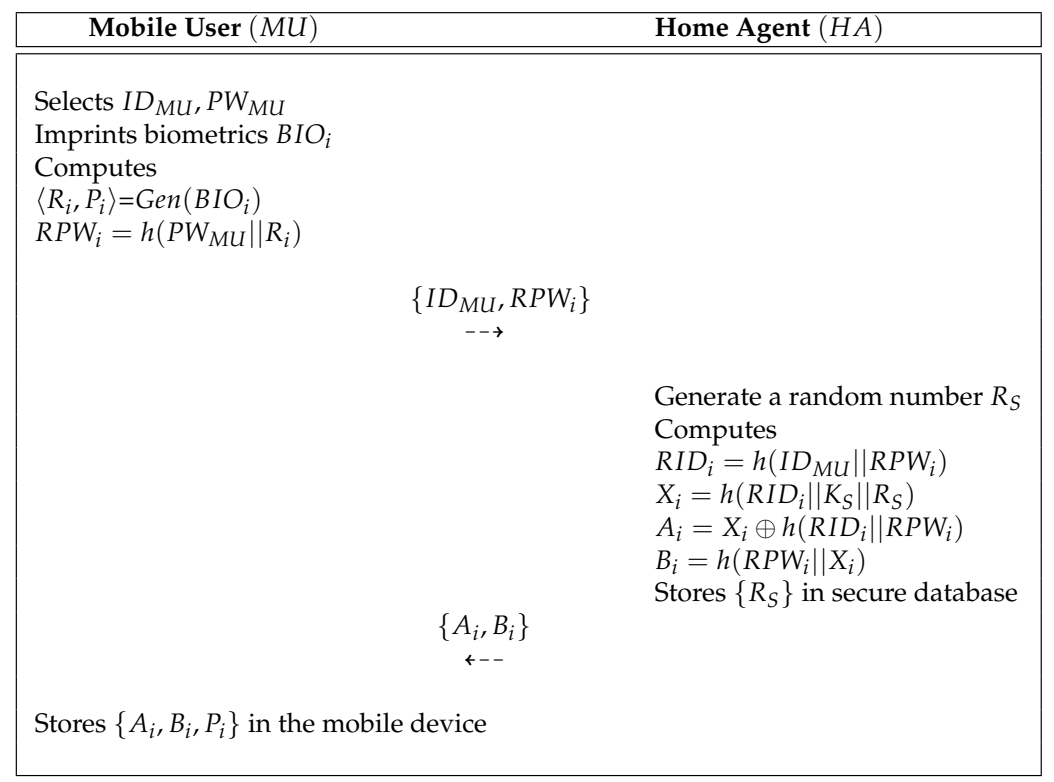

Figure 3. The user registration process of the proposed protocol.

Step 1: A $M U$ selects $I D_{M U}, P W_{M U}$ and imprints biometric $B I O_{i}$. After that, $M U$ computes $\left\langle R_{i}, P_{i}\right\rangle=\operatorname{Gen}\left(B I O_{i}\right), R P W_{i}=h\left(P W_{M U} \| R_{i}\right)$ and sends $\left\{I D_{M U}, R P W_{i}\right\}$ to the $H A$ over a secure communication.

Step 2: After obtaining messages $\left\{I D_{M U}, R P W_{i}\right\}$, the $H A$ computes $R I D_{i}=h\left(I D_{M U} \| R P W_{i}\right)$, $X_{i}=h\left(R I D_{i}\left\|K_{S}\right\| R_{S}\right), A_{i}=X_{i} \oplus h\left(R I D_{i} \| R P W_{i}\right)$ and $B_{i}=h\left(R P W_{i} \| X_{i}\right)$. After that, 
$H A$ stores $\left\{R_{S}\right\}$ in a secure database. Finally, the $H A$ sends $\left\{A_{i}, B_{i}\right\}$ to the $M U$ via a secure communication.

Step 3: Upon reception of $\left\{A_{i}, B_{i}\right\}$, the $M U$ stores $\left\{A_{i}, B_{i}, P_{i}\right\}$ in the mobile device.

\subsection{Login and Authentication Process}

Before performing a session, the $M U$ requests authentication to the $H A$ in order to establish the session key. Figure 4 presents the user authentication process of our protocol. The process is described in detail as follow.

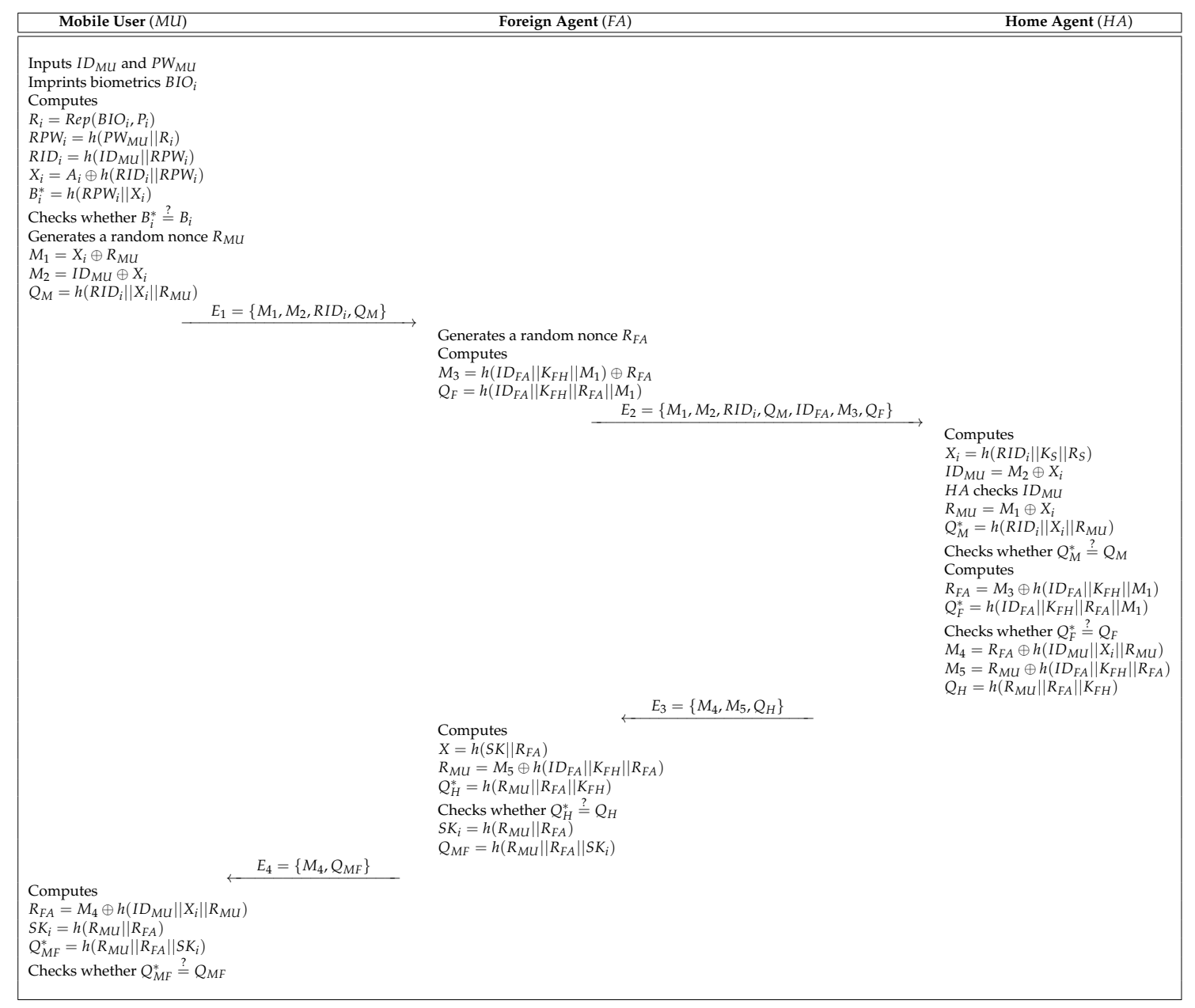

Figure 4. Login and authentication process of the proposed protocol.

Step 1: The mobile device inputs $I D_{M U}, P W_{M U}$ and imprints biometrics $B I O_{i}$. The $M U$ computes $R_{i}=\operatorname{Rep}\left(B I O_{i}, P_{i}\right), R P W_{i}=h\left(P W_{M U} \| R_{i}\right), R I D_{i}=h\left(I D_{M U} \| R P W_{i}\right), X_{i}=A_{i} \oplus$ $h\left(R I D_{i} \| R P W_{i}\right)$, and $B_{i}^{*}=h\left(R P W_{i} \| X_{i}\right)$ and checks whether $B^{*} \stackrel{?}{=} B_{i}$. If this holds, the $M U$ generates a random nonce $R_{M U}$ and computes $M_{1}=X_{i} \oplus R_{M U}, M_{2}=I D_{M U} \oplus X_{i}$ and $Q_{M}=h\left(R I D_{i}\left\|X_{i}\right\| R_{M U}\right)$. After that, $M U$ sends $\left\{E_{1}\right\}$ to the $F A$ over an open channel.

Step 2: Upon reception of $E_{1}$, the $F A$ selects a random nonce $R_{F A}$ and computes $M_{3}=$ $h\left(I D_{F A}|| K_{F H}|| M_{1}\right) \oplus R_{F A}, Q_{F}=h\left(I D_{F A}|| K_{F H}|| R_{F A}|| M_{1}\right)$. After that, the $F A$ sends $\left\{E_{2}\right\}$ to the $H A$.

Step 3: Upon reception of $E_{2}$, the $H A$ computes $X_{i}=h\left(R I D_{i}\left\|K_{S}\right\| R_{S}\right), I D_{M U}=M_{2} \oplus X_{i}$ and checks the identity $I D_{M U}$ of the mobile user. Then, $H A$ computes $R_{M U}=M_{1} \oplus X_{i}, Q_{M}^{*}=$ $h\left(R I D_{i}\left\|X_{i}\right\| R_{M U}\right)$ and checks whether $Q_{M}^{*} \stackrel{?}{=} Q_{M}$. If it is valid, the $H A$ calculates $R_{F A}=$ $M_{3} \oplus h\left(I D_{F A} \| K_{F H}|| M_{1}\right), Q_{F}^{*}=h\left(I D_{F A}\left\|K_{F H}|| R_{F A}\right\| M_{1}\right)$ and checks whether $Q_{F}^{*} \stackrel{?}{=} Q_{F}$. Then, 
the $H A$ computes $M_{4}=R_{F A} \oplus h\left(R I D_{i} \| X_{i}|| R_{M U}\right), M_{5}=R_{M U} \oplus h\left(I D_{F A}\left\|K_{F H}\right\| R_{F A}\right)$ and $Q_{H}=h\left(R_{M U}\left\|R_{F A}\right\| K_{F H}\right)$. Finally, the $H A$ sends an authentication message $\left\{E_{3}\right\}$ to the $F A$.

Step 4: Upon reception of $E_{3}$, the $F A$ computes $R_{M U}=M_{5} \oplus h\left(I D_{F A} \| K_{F H}|| R_{F A}\right), Q_{H}^{*}=$ $h\left(R_{M U}\left\|R_{F A}\right\| K_{F H}\right)$ and checks whether $Q_{H}^{*} \stackrel{?}{=} Q_{H}$. If it is correct, the $F A$ computes $S K_{i}=h\left(R_{M U} \| R_{F A}\right), Q_{M F}=h\left(R_{M U}\left\|R_{F A}\right\| S K_{i}\right)$ and sends $\left\{E_{4}\right\}$ to the $M U$.

Step 5: Upon reception of $E_{4}$, the $M U$ calculates $R_{F A}=M_{4} \oplus h\left(R I D_{i}\left\|X_{i}\right\| R_{M U}\right), S K_{i}=$ $h\left(R_{M U} \| R_{F A}\right)$, and $Q_{M F}^{*}=h\left(R_{M U}\left\|R_{F A}\right\| S K_{i}\right)$. Finally, the $M U$ checks whether $Q_{M F}^{*} \stackrel{?}{=} Q_{M F}$. If it holds, the $M U$ and $F A$ establish the $S K_{i}$ successfully.

\subsection{Password Update Process}

In the proposed protocol, a $M U$ can easily update their password. Figure 5 presents the password change process of the proposed protocol.

\begin{tabular}{|c|c|c|}
\hline Mobile User $(M U)$ & & Mobile Device \\
\hline $\begin{array}{l}\text { Inputs } I D_{M U}^{*}, P W_{M U}^{*} \\
\text { Imprints biometrics } B I O_{i}^{*} \\
\text { Computes } \\
\left\langle R_{i}, P_{i}\right\rangle=\operatorname{Gen}\left(B I O_{i}^{*}\right) \\
R P W_{i}^{*}=h\left(P W_{M U} \| R_{i}\right)\end{array}$ & $\begin{array}{c}\left\{I D_{M U^{*}}^{*}, R P W_{i}^{*}\right\} \\
-\rightarrow \\
\left\{\begin{array}{c}\text { Authenticate }\} \\
\leftarrow--\end{array}\right. \\
\left\{R P W_{i}^{\text {new }}\right\} \\
-\rightarrow\end{array}$ & $\begin{array}{l}\text { Computes } \\
A_{i}^{\text {new }}=X_{i}^{*} \oplus h\left(R I D_{i}^{*} \| R P W_{i}^{\text {new }}\right) \\
B_{i}^{\text {new }}=h\left(R P W_{i}^{\text {new }} \| X_{i}^{*}\right) \\
\text { Replaces }\left\{A_{i}, B_{i}\right\} \text { with }\left\{A_{i}^{\text {new }}, B_{i}^{\text {new }}\right\}\end{array}$ \\
\hline
\end{tabular}

Figure 5. Password change process of the proposed protocol.

Step 1: The $M U$ inputs $I D_{M U}^{*}, P W_{M U}^{*}$ and imprints biometrics $B I O_{i}^{*}$. After that, $M U$ computes $\left\langle R_{i}, P_{i}\right\rangle=\operatorname{Gen}\left(B I O_{i}^{*}\right), R P W_{i}^{*}=h\left(P W_{M U}|| R_{i}\right)$ and sends $\left\{I D_{M U}^{*}, R P W_{i}^{*}\right\}$ to the mobile device.

Step 2: Upon reception of $\left\{I D_{M U}^{*}, R P W_{i}^{*}\right\}$, the mobile device computes $R I D_{i}^{*}=h\left(I D_{M U}^{*} \| R P W_{i}^{*}\right)$, $X_{i}^{*}=h\left(R I D_{i}^{*} \| R P W_{i}^{*}\right) \oplus A_{i}, B_{i}^{*}=h\left(R P W_{i}^{*} \| X_{i}^{*}\right)$, and the mobile device checks whether $B_{i}^{*} \stackrel{?}{=} B_{i}$. If it is correct, the mobile device sends the authentication response message to the $M U$.

Step 3: Upon reception of the authentication response message, the $M U$ inputs a new password $P W_{i}^{\text {new }}$ and imprints a new biometrics $B I O_{i}^{\text {new }} . M U$ computes $\left\langle R_{i}^{\text {new }}, P_{i}^{\text {new }}\right\rangle=\operatorname{Gen}\left(B I O_{i}^{\text {new }}\right)$, $R P W_{i}^{\text {new }}=h\left(P W_{i}^{\text {new }}|| R_{i}^{\text {new }}\right)$ and sends $\left\{R P W_{i}^{\text {new }}\right\}$ to the mobile device.

Step 4: Upon reception of $\left\{R P W_{i}^{\text {new }}\right\}$, the mobile device computes $A_{i}^{\text {new }}=X_{i}^{*} \oplus h\left(R I D_{i}^{*} \| R P W_{i}^{\text {new }}\right)$, $B_{i}^{\text {new }}=h\left(R P W_{i}^{\text {new }} \| X_{i}^{*}\right)$ and replaces $\left\{A_{i}, B_{i}\right\}$ with $\left\{A_{i}^{\text {new }}, B_{i}^{\text {new }}\right\}$.

\section{Security Analysis}

We utilized the BAN logic to evaluate the user authentication of our protocol and then we used the ROR model to prove the session key security. In addition, we performed AVISPA simulation to evaluate the security of our protocol to replay and MITM attacks. 


\subsection{Informal Security Analysis}

This section presents an informal security analysis to evaluate the security of the proposed protocol. We proved that our scheme can prevent various attacks and allow user authentication and anonymity.

\subsubsection{Masquerade Attack}

If a $M U_{a}$ attempts to impersonate a legal mobile user, $M U_{a}$ must calculate a request message $\left\{M_{1}, M_{2}, R I D_{i}, Q_{M}\right\}$ and response message $\left\{M_{4}, Q_{M F}\right\}$ successfully. However, $M U_{a}$ cannot compute this because $M U_{a}$ does not know $M U$ 's real identity $I D_{M U}$, password $P W_{M U}$, secret parameters $X_{i}$, random nonce $R_{M U}$, and biometrics $B I O_{i}$. Consequently, the proposed protocol can withstand masquerade attacks because $M U_{a}$ cannot generate correct messages successfully.

\subsubsection{Replay Attack}

Our protocol can resist replay attacks utilizing random nonce that is changed every session. If a $M U_{a}$ may try to impersonate a mobile user by resending messages that were exchanged in a previous session, $M U_{a}$ cannot obtain the previous messages because the $H A$ checks whether $R_{M U}^{*} \stackrel{?}{=}$ $R_{M U}$ and $R_{F A}^{*} \stackrel{?}{=} R_{F A}$. Consequently, the proposed protocol can withstand replay attacks because $M U_{a}$ does not know $R_{M U}$ and $R_{F A}$.

\subsubsection{Stolen Mobile Device Attack}

We assume that a $M U_{a}$ can steal the mobile device of a legitimate user and extract the data $\left\{A_{i}, B_{i}, P_{i}\right\}$ from the mobile device by utilizing a power analysis attack [20]. However, $M U_{a}$ still cannot obtain a legitimate user's information because the parameters stored in the mobile device are masked using bitwise XOR operations and hash functions. Thus, the proposed scheme can defend against mobile device theft attacks.

\subsubsection{Session Key Disclosure Attack}

In our protocol, a $M U_{a}$ cannot compute $\left\{M_{1}, M_{2}, Q_{M}\right\}$ because a legitimate mobile user $M U$ generates an authentication request message by using the dynamic random nonce $R_{M U}$ and secret parameter $X_{i}$. Consequently, the proposed protocol protects against session key disclosure attacks.

\subsubsection{Anonymity}

In our protocol, a $M U_{a}$ cannot obtain the identity $I D_{M U}$ of a legitimate mobile user because the parameters are masked by using XOR operations and hash functions, such as $M_{2}=I D_{M U} \oplus X_{i}$ and $Q_{M}=h\left(R I D_{i}\left\|X_{i}\right\| R_{M U}\right)$. Consequently, our protocol provides anonymity because a $M U_{a}$ cannot obtain $I D_{M U}$ without $X_{i}$ and $R_{M U}$.

\subsubsection{Mutual Authentication}

After obtaining the login request messages $\left\{M_{1}, M_{2}, R I D_{i}, Q_{M}\right\}$ from $M U$, the $H A$ checks whether $Q_{M}^{*} \stackrel{?}{=} Q_{M}$. If this holds, $H A$ authenticates $M U$. After obtaining the messages $\left\{M_{3}, Q_{F}\right\}$ from $F A$, the $H A$ checks whether $Q_{F}^{*} \stackrel{?}{=} Q_{F}$. If it is valid, $H A$ authenticates $F A$. After obtaining the messages $\left\{M_{4}, M_{5}, Q_{H}\right\}$ from $H A, F A$ checks whether $Q_{H}^{*} \stackrel{?}{=} Q_{H}$. If this holds, $F A$ authenticates $H A$. Finally, $M U$ checks whether $Q_{M F}^{*} \stackrel{?}{=} Q_{M F}$. If this holds, $M U$ authenticates $H A$. Consequently, our protocol ensures secure mutual authentication among $M U, F A$ and $H A$ because a $M U_{a}$ does not know the secret parameter of $M U$ and $F A$. 


\subsubsection{User Friendliness}

In our protocol, $M U$ can easily change his/her own $I D_{i}$ and $P W_{i}$ without the assistance of the $H A$. In particular, the proposed protocol allows the $M U$ to change the original password $P W_{i}$ in a short time. Because, the $M U$ need not go through the entire login process, which saves the time as well as minimizes the computation complexity of the proposed scheme. Consequently, the proposed protocol is user-friendly.

\subsection{Security Properties}

Table 3 presents the better security properties ensured by the proposed scheme compared to related schemes $[6,8,15,16]$. The existing schemes are insecure various attacks and their scheme cannot ensure mutual authentication and user anonymity. In contrast, the proposed scheme can provide essential security properties and can achieve user anonymity and mutual authentication.

Table 3. Security features compared to existing schemes.

\begin{tabular}{|c|c|c|c|c|c|}
\hline Security features & He et al. [6] & Kuo et al. [8] & Karuppiah et al. [15] & Madhusudhan et al. [16] & Ours \\
\hline User anonymity & $\times$ & $\times$ & $\circ$ & $\circ$ & $\circ$ \\
\hline User friendliness & $\circ$ & $\circ$ & $\circ$ & $\circ$ & $\circ$ \\
\hline Mutual authentication & $\times$ & $\circ$ & $\circ$ & $\times$ & $\circ$ \\
\hline Insider attack & $\circ$ & $\circ$ & $\circ$ & $\circ$ & $\circ$ \\
\hline Replay attack & $\circ$ & $\times$ & $\circ$ & $\times$ & $\circ$ \\
\hline Perfect forward secrecy attack & $\circ$ & $\circ$ & $\circ$ & $\circ$ & $\circ$ \\
\hline Session key disclosure attack & $\times$ & $x$ & $\circ$ & $\times$ & $\circ$ \\
\hline Masquerade attack & $\times$ & $\circ$ & $\circ$ & $\times$ & $\circ$ \\
\hline
\end{tabular}

$\circ:$ it supports the security feature; $x$ : it does not support the security feature.

\subsection{Authentication Proof Using BAN Logic}

We present the security analysis utilizing the BAN logic [25] to prove the secure user authentication of our protocol. In Table 4, we present the notations used for BAN logic. We present the security rules, the security goals, the idealized forms and the assumptions that are essential to BAN logic. We assessed that our scheme ensured mutual authentication among $M U, F A$, and $H A$.

Table 4. Notations used for BAN logic.

\begin{tabular}{cc}
\hline Notation & Description \\
\hline$A \mid \equiv B$ & $A$ believes that $B$ \\
\hline$\# B$ & $B$ is updated and fresh \\
\hline$A \triangleleft B$ & $A$ sees that $B$ \\
\hline$A \mid \sim B$ & $A$ once said $B$ \\
\hline$A \Rightarrow B$ & $A$ controls that $B$ \\
\hline$<B>_{W}$ & $B$ is combined with $W$ \\
\hline$\{B\}_{K}$ & $B$ is encrypted utilizing symmetric key $K$ \\
\hline$A \stackrel{\leftrightarrow}{\leftrightarrow} P$ & $A$ and $P$ can make secure contact utilizing $K$ as the shared secret key \\
\hline$S K$ & Session key used in communication session \\
\hline
\end{tabular}

\subsubsection{Rules of BAN Logic}

The rules of BAN logic are summarized as follows. 
1. Message meaning rule :

$$
\frac{A \mid \equiv A \stackrel{K}{\leftrightarrow} P, \quad A \triangleleft\{B\}_{K}}{A|\equiv P| \sim B}
$$

2. Nonce verification rule :

$$
\frac{A|\equiv \#(B), \quad A| \equiv P \mid \sim B}{A|\equiv P| \equiv B}
$$

3. Jurisdiction rule :

$$
\frac{A|\equiv P| \Longrightarrow B, \quad A|\equiv P| \equiv B}{A \mid \equiv B}
$$

4. Freshness rule :

$$
\frac{A \mid \equiv \#(B)}{A \mid \equiv \#(B, W)}
$$

5. Belief rule :

$$
\frac{A \mid \equiv(B, W)}{A \mid \equiv B}
$$

\subsubsection{Goals}

To analyze mutual authentication, we define the goals of our protocol as below.

Goal 1: $\quad M U \mid \equiv(M U \stackrel{S K}{\longleftrightarrow} F A)$

Goal 2: $F A \mid \equiv(M U \stackrel{S K}{\longleftrightarrow} F A)$

Goal 3: $\quad M U|\equiv F A| \equiv(M U \stackrel{S K}{\longleftrightarrow} F A)$

Goal 4: $F A|\equiv M U| \equiv(M U \stackrel{S K}{\longleftrightarrow} F A)$.

\subsubsection{Idealized Forms}

The idealized form of messages of our protocol are as below.

$$
\begin{array}{ll}
M s g_{1}: & M U \rightarrow F A:\left(R I D_{i}, I D_{M U}, R_{M U}\right)_{X_{i}} \\
M s g_{2}: & F A \rightarrow H A:\left(R I D_{i}, I D_{M U}, R_{M U}, X_{i}, R_{F A}, I D_{F A}\right)_{K_{F H}} \\
M s g_{3}: & H A \rightarrow F A:\left(I D_{M U}, I D_{F A}, R_{F A}, R_{M U}\right)_{K_{F H}} \\
M s g_{4}: & F A \rightarrow M U:\left(I D_{M U}, R_{M U}, R_{F A},(M U \stackrel{S K}{\longleftrightarrow} F A)\right)_{X_{i}} .
\end{array}
$$

\subsubsection{Assumptions}

The following assumptions are applied in the BAN logic analysis.

$$
\begin{array}{ll}
A_{1}: & F A \mid \equiv\left(M U \stackrel{X_{i}}{\longleftrightarrow} F A\right) \\
A_{2}: & F A \mid \equiv \#\left(R_{M U}\right) \\
A_{3}: & H A \mid \equiv\left(H A \stackrel{K_{F H}}{\longleftrightarrow} F A\right) \\
A_{4}: & H A \mid \equiv \#\left(R_{F A}\right) \\
A_{5}: & F A \mid \equiv\left(H A \stackrel{K_{F H}}{\longleftrightarrow} F A\right) \\
A_{6}: & F A \mid \equiv \#\left(R_{F A}\right) \\
A_{7}: & M U \mid \equiv\left(M U \stackrel{X_{i}}{\longleftrightarrow} F A\right) \\
A_{8}: & M U \mid \equiv \#\left(R_{F A}\right) \\
A_{9}: & M U \mid \equiv F A \Rightarrow(M U \stackrel{S K}{\longleftrightarrow} F A) \\
A_{10}: & F A \mid \equiv M U \Rightarrow(M U \stackrel{S K}{\longleftrightarrow} F A) .
\end{array}
$$




\subsubsection{Proof Using BAN Logic}

The proof then proceeds as below:

Step 1: According to $M s g_{1}$, we obtain the following

$$
\left(S_{1}\right): F A \triangleleft\left(R I D_{i}, I D_{M U}, R_{M U}\right)_{X_{i}} .
$$

Step 2: Utilizing $S_{1}$ and $A_{1}$ with the "message meaning rule", the following is obtained

$$
\left(S_{2}\right): F A|\equiv M U| \sim\left(R I D_{i}, I D_{M U}, R_{M U}\right)_{X_{i}} .
$$

Step 3: Now, using $S_{2}$ and $A_{2}$ with the "freshness rule", the following is obtained

$$
\left(S_{3}\right): F A \mid \equiv \#\left(R I D_{i}, I D_{M U}, R_{M U}\right)_{X_{i}} .
$$

Step 4: Utilizing $S_{2}$ and $S_{3}$ with the "nonce verification rule", we obtain

$$
\left(S_{4}\right): F A|\equiv M U| \equiv\left(R I D_{i}, I D_{M U}, R_{M U}\right)_{X_{i}} .
$$

Step 5: Utilizing $S_{4}$ and the "belief rule", we obtain

$$
\left(S_{5}\right): F A|\equiv M U| \equiv\left(R_{M U}\right)_{X_{i}} .
$$

Step 6: According to $M s g_{2}$, we obtain

$$
\left(S_{6}\right): H A \triangleleft\left(R I D_{i}, I D_{M U}, R_{M U}, X_{i}, R_{F A}, I D_{F A}\right)_{K_{F H}} .
$$

Step 7: Utilizing the $S_{6}$ and $A_{3}$ with the "message meaning rule", the following is obtained

$$
\left(S_{7}\right): H A|\equiv F A| \sim\left(R I D_{i}, I D_{M U}, R_{M U}, X_{i}, R_{F A}, I D_{F A}\right)_{K_{F H}} .
$$

Step 8: Now, using $S_{7}$ and $A_{4}$ with the "freshness rule", we obtain

$$
\left(S_{8}\right): H A \mid \equiv \#\left(R I D_{i}, I D_{M U}, R_{M U}, X_{i}, R_{F A}, I D_{F A}\right)_{K_{F H}} .
$$

Step 9: Utilizing $S_{7}$ and $S_{8}$ with the "nonce verification rule", the following is obtained

$$
\left(S_{9}\right): H A|\equiv F A| \equiv\left(R I D_{i}, I D_{M U}, R_{M U}, X_{i}, R_{F A}, I D_{F A}\right)_{K_{F H}} .
$$

Step 10: According to $\mathrm{Msg}_{3}$, we obtain

$$
\left(S_{10}\right): F A \triangleleft\left(I D_{F A}, R_{F A}, R_{M U}\right)_{K_{F H}} .
$$

Step 11: Utilizing $S_{10}$ and $A_{5}$ with the "message meaning rule", the following is obtained

$$
\left(S_{11}\right): F A|\equiv H A| \sim\left(I D_{F A}, R_{F A}, R_{M U}\right)_{K_{F H}} .
$$

Step 12: Now, using $S_{11}$ and $A_{6}$ with the "freshness rule", we obtain

$$
\left(S_{12}\right): F A \mid \equiv \#\left(I D_{F A}, R_{F A}, R_{M U}\right)_{K_{F H}} .
$$

Step 13: Utilizing $S_{11}$ and $S_{12}$ with the "nonce verification rule", the following is obtained

$$
\left(S_{13}\right): F A|\equiv H A| \equiv\left(I D_{F A}, R_{F A}, R_{M U}\right)_{K_{F H}} .
$$


Step 14: According to $M s g_{4}$, we could obtain

$$
\left(S_{14}\right): M U \triangleleft\left(I D_{M U}, R_{M U}, R_{F A},(M U \stackrel{S K}{\longleftrightarrow} F A)\right)_{X_{i}}
$$

Step 15: Utilizing $S_{14}$ and $A_{7}$ with the "message meaning rule", we obtain

$$
\left(S_{15}\right): M U|\equiv F A| \sim\left(I D_{M U}, R_{M U}, R_{F A},(M U \stackrel{S K}{\longleftrightarrow} F A)\right)_{X_{i}} .
$$

Step 16: Now, using $S_{15}$ and $A_{8}$ with the "freshness rule", the following is obtained

$$
\left(S_{16}\right): M U \mid \equiv \#\left(I D_{M U}, R_{M U}, R_{F A},(M U \stackrel{S K}{\longleftrightarrow} F A)\right)_{X_{i}} \cdot
$$

Step 17: Utilizing $S_{15}$ and $S_{16}$ with the "nonce verification", we obtain

$$
\left(S_{17}\right): M U|\equiv F A| \equiv\left(I D_{M U}, R_{M U}, R_{F A},(M U \stackrel{S K}{\longleftrightarrow} F A)\right)_{X_{i}} \cdot
$$

Step 18: Utilizing $S_{17}$ and the belief rule, we obtain

$$
\left(S_{18}\right): M U|\equiv F A| \equiv(M U \stackrel{S K}{\longleftrightarrow} F A) . \quad \text { (Goal 3) }
$$

Step 19: Now, using $S_{18}$ and $A_{9}$ with the "jurisdiction rule", the following is obtained

$$
\left(S_{19}\right): M U \mid \equiv(M U \stackrel{S K}{\longleftrightarrow} F A) . \quad \text { (Goal 1) }
$$

Step 20: Because of $S K=h\left(R_{M U} \| R_{F A}\right)$, from the $S_{5}, S_{9}, S_{13}$ and $S_{17}$ we obtain

$$
\left(S_{20}\right): F A|\equiv M U| \equiv(M U \stackrel{S K}{\longleftrightarrow} F A) . \quad \text { (Goal 4) }
$$

Step 21: Utilizing $S_{19}$ and $A_{10}$ with the "jurisdiction rule", we obtain

$$
\left(S_{21}\right): F A \mid \equiv(M U \stackrel{S K}{\longleftrightarrow} F A) . \quad \text { (Goal 2) }
$$

Based on goals 1 to 4 , we proved that $M U, F A$, and $H A$ are securely mutually authenticated. We assessed that the proposed scheme ensured mutual authentication between $M U, F A$, and $H A$.

\subsection{ROR Model Analysis}

To evaluate the session key (SK) security of the protocol from the malicious adversary $U_{A}$, the proposed protocol performs the ROR model [26], which is a widely known formal security analysis. We first introduce the ROR model before doing a SK security proof for the proposed protocol.

Participants: There are three participants: the mobile user $P_{M U}^{t_{1}}$, the foreign agent $P_{F A}^{t_{2}}$, and the home agent $P_{H A}^{t_{3}}$ are instances $t_{1}^{\text {th }}$ of the $M U, t_{2}^{\text {th }}$ of the $F A$, and $t_{3}^{t h}$ of the $H A$, respectively.

Partnering: The instances $t_{1}^{\text {th }}$ and $t_{2}^{\text {th }}$ are partners if they satisfy the following conditions: (1) $t_{1}^{\text {th }}$ and $t_{2}^{\text {th }}$ are in the accept state, (2) $t_{1}^{\text {th }}$ and $t_{2}^{\text {th }}$ authenticate each other mutually sharing the same sid, and (3) $t_{1}^{\text {th }}$ and $t_{2}^{\text {th }}$ are mutually authenticated.

Freshness: If the $U_{A}$ does not obtain the SK between $M U$ and $F A$ by utilizing the reveal query Reveal, the instance $t_{1}^{\text {th }}$ or $t_{2}^{\text {th }}$ is considered fresh.

Adversary: In the ROR model, the $U_{A}$ can eavesdrop, modify, delete, or insert the exchanged messages during the communication. Furthermore, the $U_{A}$ will have the access to the following queries.

- $\operatorname{Execute}\left(P_{M U}^{t_{1}}, P_{F A}^{t_{2}}, P_{H A}^{t_{3}}\right)$ : It denotes that $U_{A}$ performs the eavesdropping attack by eavesdropping exchanged messages between $M U, F A$, and $H A$ over wireless communication.

- CorruptDevice $\left(P_{M U}^{t_{1}}\right)$ : It is modeled from the mobile device lost/stolen attack, in which the $U_{A}$ is able to extract the secret data in the mobile device. 
- $\quad \operatorname{Send}\left(P^{t}, M\right)$ : In this query, the $U_{A}$ can dispatch a message $M$ to the instance $P^{t}$ and can also reply accordingly.

- $\quad \operatorname{Test}\left(P^{t}\right)$ : It corresponds to the semantic security of the $S K_{i j}$ between $M U$ and $F A$ following the indistinguishability style in the ROR model [26]. In this query, before the experiment starts, an unbiased coin $c$ is tossed. If the $U_{A}$ executes Tset query and the established $S K_{i j}$ is fresh, then $P^{t}$ returns $S K_{i j}$ for the case when $c=1$ or a random value when $c=0$. On the other cases, it returns a null value $(\perp)$.

- Reveal $\left(P^{t}\right)$ : With this query, the $U_{A}$ can reveal the $S K_{i}$ created by its partner to $U_{A}$ in the current session.

Semantic security of the session key: In this formal security model, the malicious adversary $U_{A}$ must distinguish between an instance's actual $S K$ and a random secret key. The $U_{A}$ can perform Test queries to either $P_{M U}^{t_{1}}$ or $P_{F A}^{t_{2}}$, and its output is checked for consistency against the random bit c. If the condition $c^{\prime}=c$ is valid, the $U_{A}$ wins the game. Otherwise, the $U_{A}$ loses the game. Let Succ denote an event that is $U_{A}$ winning the game. Therefore, the advantage of $U_{A}$ in breaking the semantic security of our protocol $P$ is shown in Equation (1). The proposed protocol $P$ is secure relative to the ROR model when $A d v_{P} \leq \psi$, for any sufficiently small $\psi>0$.

$$
A d v_{P}=|2 \cdot \operatorname{Pr}[S u c c]-1|
$$

Random oracle: In this paper, all the participants and the malicious adversary $U_{A}$ can access a collision-resistant one-way hash function $h(\cdot)$. We model $h(\cdot)$ as a random oracle, say Hash.

\subsubsection{Security Proof}

We utilized Zipf's law [27] to assess the SK security of our protocol and the detailed theorems are given as follows:

Theorem 1. If $A d v_{U_{A}}$ denotes the advantage function of the $U_{A}$ in violating $S K$ security of our protocol. Then, we obtain the following.

$$
A d v_{U_{A}} \leq \frac{q_{h}^{2}}{\mid \text { Hash } \mid}+2\left\{C \cdot q_{\text {send }}^{s}, \frac{q_{s}}{2^{l_{b}}}\right\}
$$

where Hash, $q_{\text {send }}$, and $q_{h}$ are the number of Hash queries, the number of Send queries, and the range space of the hash function $h($.$) , respectively; l_{b}$ is the number of bits present in the $M U_{i}$ 's biometric secret key $b_{i}$; and $s$ and $C$ are the Zipf's parameters [27].

Proof. We follow the proof as presented in [28,29]. A sequence of five games denoted by $G M_{i}$, where $i \in[0,3]$, are defined to demonstrate the SK security of our protocol. Succ $i$ denotes the probability of $U_{A}$ winning the game $G M_{i}$. Each game is described in detail as follows.

- Game $G M_{0}$ : This game is considered as an actual attack by the $U_{A}$ for the proposed protocol $P$. Since the bit $c$ is guessed at the beginning of $G_{0}$. According to this game, we obtain the following:

$$
A d v_{P}=\left|2 \cdot \operatorname{Pr}\left[\operatorname{Succ}_{0}\right]-1\right| .
$$

- Game $G M_{1}$ : This game is modeled so that the $U_{A}$ performs an eavesdropping attack in which the exchanged messages $\left\{M_{1}, M_{2}, R I D_{i}, Q_{M}\right\},\left\{M_{1}, R I D_{i}, Q_{M}, I D_{F A}, M_{3}, Q_{F}\right\},\left\{M_{4}, M_{5}, Q_{H}\right\}$, and $\left\{M_{4}, Q_{M F}\right\}$ are intercepted during the authentication phase using the Execute $\left(P_{M U}^{t_{1}}, P_{F A}^{t_{2}}, P_{H A}^{t_{3}}\right)$ query. Then, $U_{A}$ performs the Test query to check whether it is the real $S K$ or a random number. In the proposed protocol, the $S K_{i}$ is calculated as $S K_{i}=h\left(R_{M U} \| R_{F A}\right)$. To derive $S K_{i}$, the $U_{A}$ needs secret credentials, such as $R_{M U}, R_{F A}$, and $X_{i}$. Consequently, the $U_{A}$ 's probability in winning $G M_{1}$ by eavesdropping on the exchanged messages does not increase. We can obtain

$$
\operatorname{Pr}\left[\text { Succ }_{1}\right]=\operatorname{Pr}\left[\text { Succ }_{0}\right] \text {. }
$$


- Game $G M_{2}$ : The difference between $G M_{1}$ and $G M_{2}$ is that the Hash and Send queries are included in $G M_{2}$. This game can be considered as an active attack in which the $U_{A}$ may try to fool a legitimate entity to accept the exchanged messages modified by the $U_{A}$. All exchanged messages are protected by using the collision-resistant one-way hash function $h(\cdot)$. All exchanged messages are constructed using the random credentials $R_{M U}, R_{F A}$, and $X_{i}$. All exchanged messages are constructed using the random credentials $R_{M U}, R_{F A}$, and $X_{i}$ and these messages are protected by using the collision-resistant one-way hash function $h($.$) . Using birthday paradox, we can obtain$ the following result:

$$
\mid \operatorname{Pr}\left[\text { Succ }_{2}\right]-\operatorname{Pr}\left[\text { Succ }_{1}\right] \mid \leq \frac{q_{h}^{2}}{2|\operatorname{Hash}|} .
$$

- Game $G M_{3}$ : In the final game, the CorruptDevice query is modeled. In this case, a $U_{A}$ can extract the secret parameters $\left\{A_{i}, B_{i}, P_{i}\right\}$ from a mobile device's memory utilizing the power-analysis attack. Here, $A_{i}=X_{i} \oplus h\left(R I D_{i} \| R P W_{i}\right), B_{i}=h\left(R P W_{i} \| X_{i}\right)$ and $P=\operatorname{Gen}\left(B I O_{i}\right)$. It is computationally infeasible for $U_{A}$ to derive the real identity $I D_{M U}$ and password $P W_{M U}$ of $M U$ correctly via the Send query without $H A^{\prime}$ 's master key $K_{s}$ and secret parameter $X_{i}$. The probability of guessing the biometric key $b_{i}$ of $l_{b}$ bits by the $U_{A}$ is approximately $\frac{1}{2^{2} b}$. Consequently, the $G M_{2}$ and $G M_{3}$ are indistinguishable if password/biometrics guessing attacks are not implemented. Therefore, utilizing Zipf's law [27], we can obtain the following result:

$$
\left|\operatorname{Pr}\left[\mathrm{Succ}_{3}\right]-\operatorname{Pr}\left[\mathrm{Succ}_{2}\right]\right| \leq \max \left\{\mathrm{C} \cdot q_{\text {send }}^{s}, \frac{q_{s}}{2^{l_{b}}}\right\}
$$

As all the games are executed, the $U_{A}$ must guess the exact bit $c$. Thus, we can obtain the following result:

$$
\operatorname{Pr}\left[\mathrm{Succ}_{3}\right]=\frac{1}{2}
$$

With Equations (1), (2), and (5), we can obtain the result as below:

$$
\begin{aligned}
\frac{1}{2} A d v_{U_{A}} & =\left|\operatorname{Pr}\left[\operatorname{Succ}_{0}\right]-\frac{1}{2}\right| \\
& =\mid \operatorname{Pr}\left[\text { Succ }_{1}\right]-\frac{1}{2} \mid \\
& =\mid \operatorname{Pr}\left[\text { Succ }_{1}\right]-\operatorname{Pr}\left[\text { Succ }_{3}\right] \mid .
\end{aligned}
$$

Using Equations (4)-(6), we can obtain the following result, which uses the triangular inequality.

$$
\begin{aligned}
\frac{1}{2} A d v_{U_{A}}= & \mid \operatorname{Pr}\left[\text { Succ }_{1}\right]-\operatorname{Pr}\left[\text { Succ }_{3}\right] \mid \\
\leq & \mid \operatorname{Pr}\left[\text { Succ }_{1}\right]-\operatorname{Pr}\left[\text { Succ }_{2}\right] \mid \\
& +\mid \operatorname{Pr}\left[\text { Succ }_{2}\right]-\operatorname{Pr}\left[\text { Succ }_{3}\right] \mid \\
\leq & \frac{q_{h}^{2}}{2 \mid \text { Hash } \mid}+\max \left\{C \cdot q_{\text {send }}^{s}, \frac{q_{s}}{2^{l_{b}}}\right\} .
\end{aligned}
$$

Finally, multiplying both sides of Equation (7) by a factor of two, we can obtain the result as belows:

$$
A d v_{U_{A}} \leq \frac{q_{h}^{2}}{|H a s h|}+2 \max \left\{C \cdot q_{\text {send }}^{s}, \frac{q_{s}}{2^{l_{b}}}\right\}
$$

\section{AVISPA Simulation}

We discuss a formal security validation of our protocol utilizing Automated Validation of Internet Security Protocols and Applications (AVISPA) [30,31], which evaluates the security of the protocol to 
MITM attacks and replay attacks. To evaluate the AVISPA, the environment and session of the protocol must be implemented utilizing the High-Level Protocols Specification Language (HLPSL).

\subsection{HLPSL Specification}

According to HLPSL, we consider three roles: the $M U$, the $F A$, and the $H A$. We define the environment and session using HLPSL in Figure 6, which comprises the security goals. Figure 7 presents the role specification of $M U$ and $F A$.

As shown in Figure 7, the $M U$ initially receives the message and changes the state value from 1 to 2 . Then, the $M U$ sends the registration request messages $\left\{I D_{M U}, R P W_{i}\right\}$ to $H A$ over a secure channel. Then, $M U$ receives the secret parameter $\left\{A_{i}, B_{i}\right\}$ from $H A$ and $M U$ updates the state value from 1 to 2 . When a $M U$ requests access to roaming services, the $M U$ must send a login request message $\left\{M_{1}, M_{2}, R I D_{i}, Q_{M}\right\}$ to $F A$ over an open channel. After that, $M U$ declares witness $\left(M U, H A, m u \_h a \_m u, R_{M U}^{\prime}\right)$ and changes the state value from 2 to 3 . Finally, $M U$ receives the message $\left\{M_{4}, Q_{M F}\right\}$ from $F A$. Then, $M U$ checks whether $Q_{M F}^{*} \stackrel{?}{=} Q_{M F}$. If this holds, the $M U$ successfully authenticates the $F A$. The role specification of $F A$ and $H A$ are similarly defined. Furthermore, Figure 8 presents the role specification of $H A$.

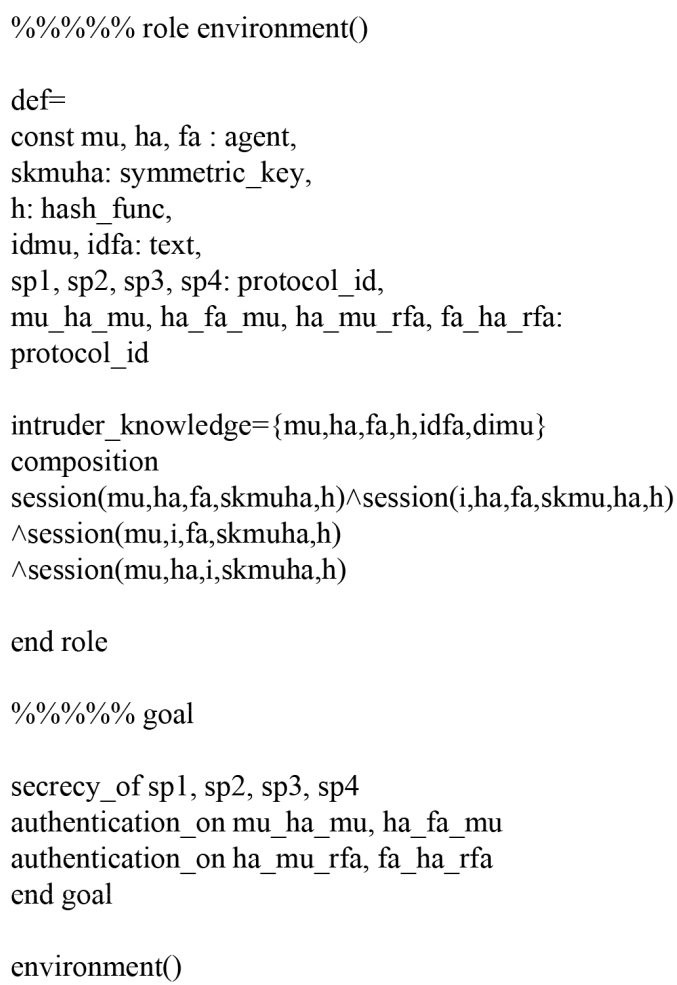

(a) Environment

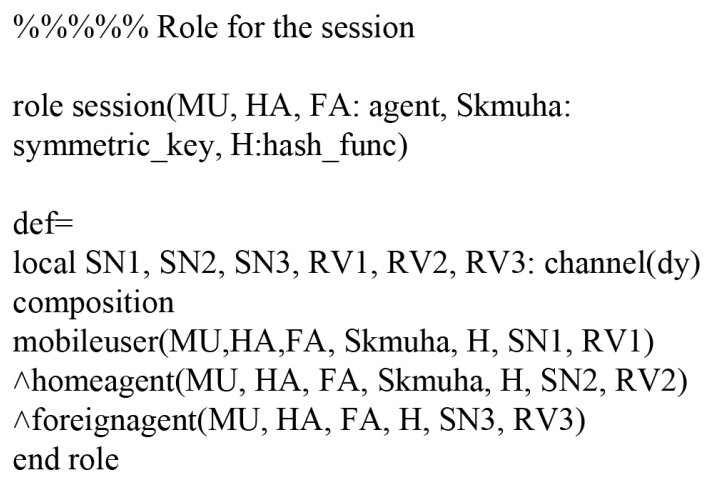

Figure 6. Role specification for the environment and session. 


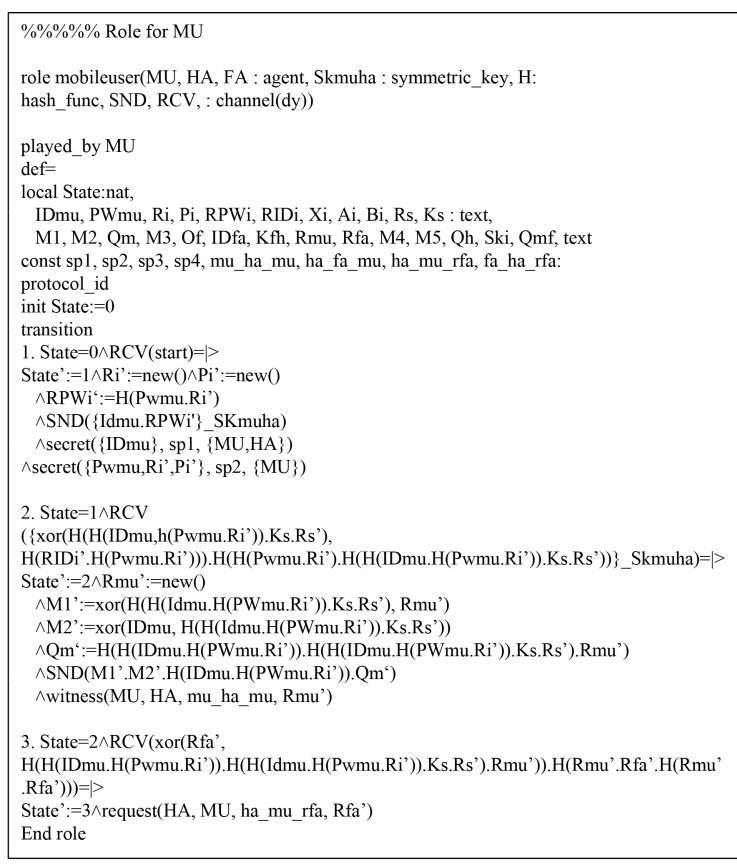

(a) Mobile user

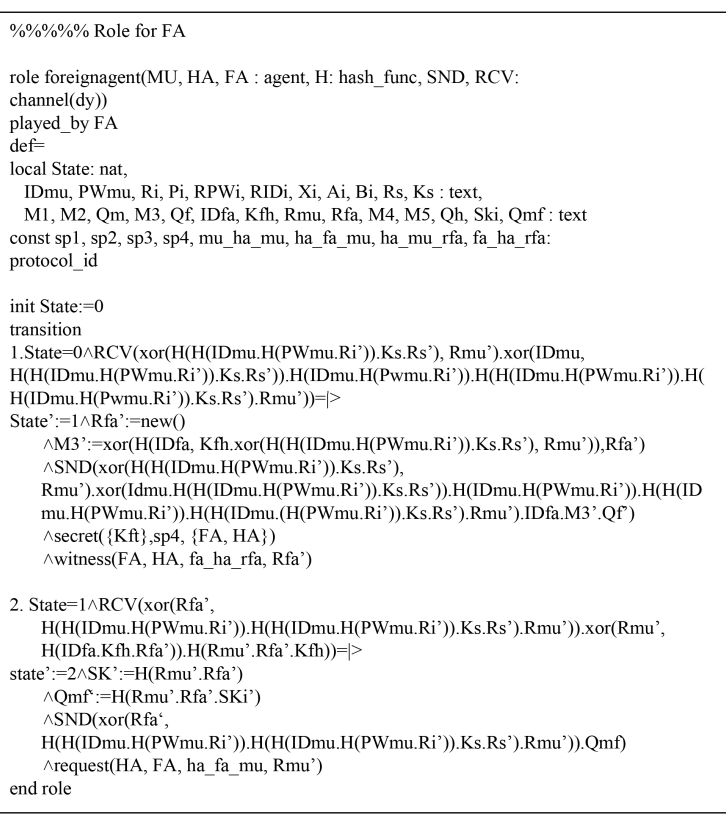

(b) Foreign agent

Figure 7. Role specification for $M U$ and $F A$.

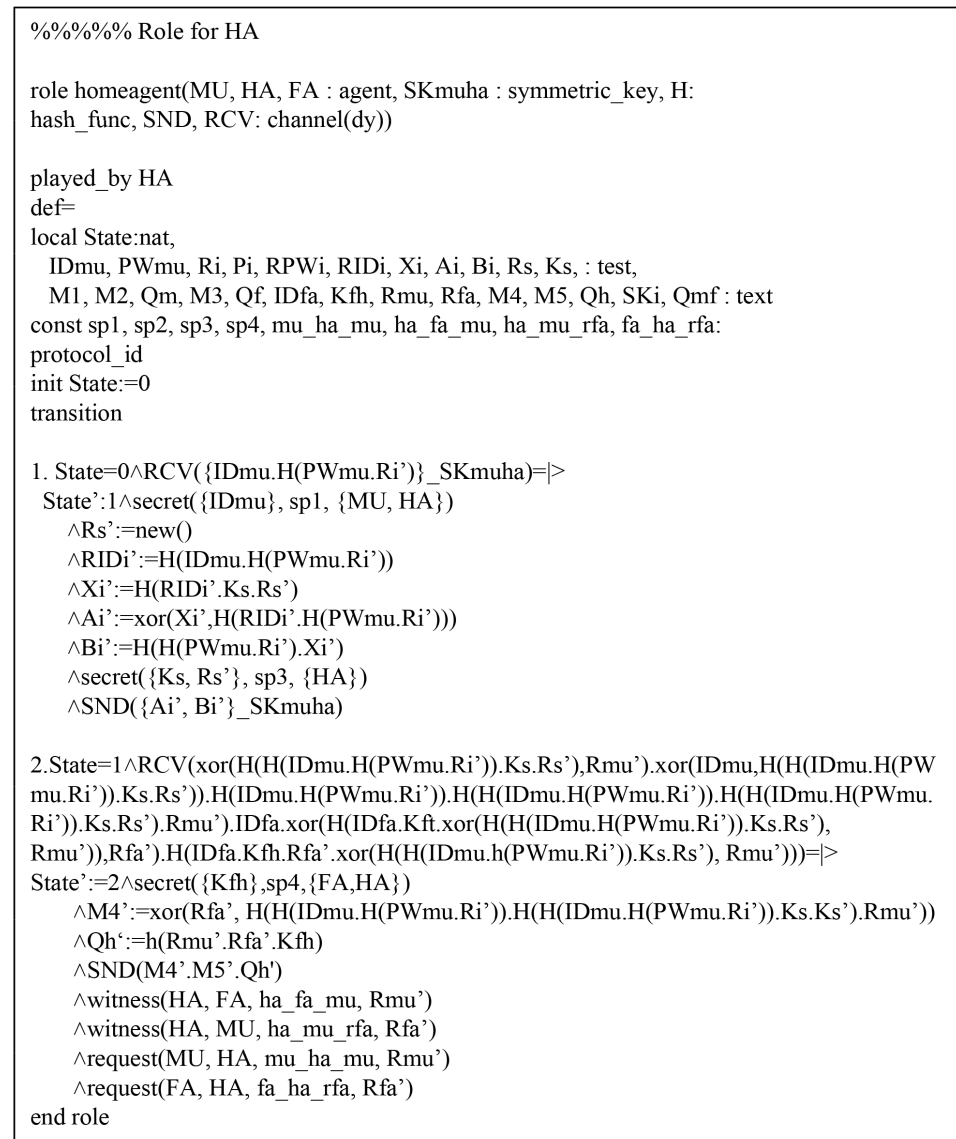

Figure 8. Role specification for $H A$. 


\subsection{Result Analysis of AVISPA Simulation}

We show the results of the AVISPA simulation using Constraint-Logic-based ATtack SEarcher (CL-AtSe) and On-the-Fly Model Checker (OFMC) to verify the security of our protocol. The CL-AtSe assessed the security of the protocol to replay attacks. The CL-AtSe verifies whether a legitimate user could perform the scheme by executing a search for a malicious adversary. Furthermore, the OFMC verifies the security of the proposed protocol to MITM attacks. The results, shown in Figure 9, demonstrate that the proposed protocol is secure against both MITM and replay attacks. The OFMC verification shows that the search time was $1.12 \mathrm{~s}$ for visiting 130 nodes, and the CL-AtSe verification analyzed three states with $0.08 \mathrm{~s}$ to translate.

\begin{tabular}{|l||l|}
\hline \multicolumn{1}{|l||}{ SUMMARY } & SUMMARY \\
SAFE & SAFE \\
& \\
DETAILS & DETAILS \\
BOUNDED_NUMBER_OF_SESSIONS & BOUNDED_NUMBER_OF_SESSIONS \\
TYPED_MODEL & PROTOCOL \\
PROTOCOL & home/span/span/testsuite/results/GLOMONET.if \\
/home/span/span/testsuite/results/GLOMONET.if & GOAL \\
GOAL & As Specified \\
As Specified & BACKEND \\
BACKEND & OFMC \\
CL-AtSe & COMMENTS \\
STATISTICS & STATISTICS \\
Analysed $: 3$ states & parseTime $: 0.00$ s \\
Reachable $: 0$ states & searchTime $: 1.12 \mathrm{~s}$ \\
Translation $: 0.08$ seconds & visitedNodes $: 130$ nodes \\
Computation $: 0.01$ seconds & depth $: 6$ plies \\
\hline
\end{tabular}

Figure 9. Analysis of the simulation results using CL-AtSe and OFMC.

\section{Performance Analysis}

This section assesses the performance of our protocol in terms of the computation cost, communication cost, and security properties. We also compared the proposed protocol with other related protocols $[6,8,15,16]$. We demonstrated that the proposed scheme provides better security properties and efficiency as compared to other related schemes.

\subsection{Computation Cost}

We compared the computation costs of our protocol to those of existing protocols $[6,8,15,16]$. Referring to [32,33], we estimated the approximate execution time of each cryptographic operation on the following configurations of the computer system. Windows 7 OS and Android phones were used and the system structure of the mobile phone ws Android 4.4.4KTU84P along with a 2 GB RAM and $1.8 \mathrm{GHz}$ processor. Furthermore, the configurations of the computer system were Windows 7 , Professional with an Intel(R) Core(TM) 2 Quad CPU Q8300, 2 GB RAM, @2.50 Hz. The XOR function was not included as it was negligible compared to other functions. The following shows the time complexity for the computational analysis.

- $\quad T_{h}$ : The time complexity of a one-way hash function operation $\approx 0.0005 \mathrm{~s}$.

- $T_{m}$ : The time complexity of a modular multiplication operation $\approx 0.00125 \mathrm{~s}$.

- $T_{m m}$ : The time complexity of a modular exponentiation operation $\approx 0.522 \mathrm{~s}$.

- $T_{p m}$ : The time complexity of a elliptic curve point multiplication operation $\approx 0.0503 \mathrm{~s}$.

- $T_{s y m}$ : The time complexity of a symmetric encryption/decryption operation $\approx 0.0087 \mathrm{~s}$.

- $T_{e c c}:$ The time complexity of a asymmetric encryption/decryption operation $\approx 0.3057 \mathrm{~s}$.

The total computation costs for our protocol and for Madhusudhan et al.'s scheme were $27 T_{h}$ $(\approx 0.0135 \mathrm{~s})$ and $10 T_{h}+3 T_{m m}+4 T_{\text {sym }}(\approx 1.6058 \mathrm{~s})$, respectively. Table 5 presents the result for 
computation costs. Consequently, we provided better efficient computation costs compared with related schemes because it only uses one-way hash functions. Therefore, the proposed scheme is considered efficient in the application for practical mobile environments.

Table 5. Computation cost comparison.

\begin{tabular}{ccccc}
\hline Schemes & Registration & Login and Authentication & Total & Total Cost (s) \\
\hline He et al. [6] & $7 T_{h}+1 T_{\text {sym }}$ & $17 T_{h}+4 T_{\text {sym }}+8 T_{\text {asym }}$ & $24 T_{h}+5 T_{\text {sym }}+8 T_{\text {asym }}$ & 2.5272 \\
\hline Kuo et al. [8] & $2 T_{h}$ & $17 T_{h}+6 T_{p m}$ & $19 T_{h}+6 T_{p m}$ & 0.3113 \\
\hline Karuppiah et al. [15] & $5 T_{h}+1 T_{\text {sym }}$ & $24 T_{h}+1 T_{m}+3 T_{m m}+3 T_{\text {sym }}$ & $29 T_{h}+1 T_{m}+3 T_{m m}+4 T_{\text {sym }}$ & 1.60785 \\
\hline Madhusudhan et al. [16] & $3 T_{h}+1 T_{m m}$ & $7 T_{h}+2 T_{m m}+4 T_{\text {sym }}$ & $10 T_{h}+3 T_{m m}+4 T_{\text {sym }}$ & 1.6058 \\
\hline Ours & $5 T_{h}$ & $22 T_{h}$ & $27 T_{h}$ & 0.0135 \\
\hline
\end{tabular}

$T_{m}$ : modular multiplication, $T_{m m}$ : modular exponentiation, $T_{h}$ : hash function, $T_{p m}$ : elliptic curve point multiplication, $T_{\text {sym }}$ : symmetric encryption/decryption, $T_{\text {asym }}$ : asymmetric encryption/decryption.

\subsection{Communication Cost}

We evaluated the communication costs of our protocol with existing schemes $[6,8,15,16]$. According to [34], we define that the identity, timestamp, and random number are 128 bits, 32 bits, and 64 bits, respectively. In addition, hash functions and symmetric key encryption require 160 bits and 256 bits, respectively. Finally, the modular operation and the scalar multiplication operation on the elliptic curve define 1024 bits and 320 bits, respectively.

Table 6 tabulates the analysis results of the communication costs. In Figure 4, the transmitted messages require $(160+160+160+160=640$ bits $),(160+160+160+160+128+160+160=1088$ bits $),(160+160+160=480$ bits $)$, and $(160+160=320$ bits $)$. Consequently, the total communication cost of our protocol was 3136 bits. Although the proposed protocol had a higher communication cost than Madhusudhan et al.'s protocol [16] and it provided better security than Madhusudhan et al.'s scheme [16].

Table 6. Communication cost comparison.

\begin{tabular}{cccc}
\hline Schemes & Registration Process & Login and Authentication Process & Total Cost \\
\hline He et al. [6] & 704 bits & 4992 bits & 5696 bits \\
\hline Kuo et al. [8] & 640 bits & 3872 bits & 4512 bits \\
\hline Karuppiah et al. [15] & 640 bits & 4224 bits & 4864 bits \\
\hline Madhusudhan et al. [16] & 1184 bits & 1344 bits & 2528 bits \\
\hline Ours & 608 bits & 2528 bits & 3136 bits \\
\hline
\end{tabular}

\section{Conclusions}

In this paper, we assessed that Madhusudhan et al.'s authentication scheme did not prevent various attacks. Furthermore, we assessed that their protocol could not achieve user authentication. We proposed a secure and efficient three-factor authentication protocol for roaming services in GLOMONET to improve the security flaws of Madhusudhan et al.'s scheme. Our scheme was able to resist various attacks, such as masquerade, replay, session key disclosure, and mobile device theft attacks and could ensure anonymity and user authentication. We demonstrated that our scheme achieved secure mutual authentication among the mobile user, the foreign agent, and the home agent by performing BAN logic analysis.

Furthermore, we assessed a formal security validation analysis of our protocol utilizing the ROR model and AVISPA simulation. We compared the computation costs and security features with existing schemes. The three-factor based proposed scheme provided a great improvement in terms of the security level compared with two-factor based existing schemes and also preserved the low 
computation cost. The principal merit of the proposed scheme was resistance against potential attacks in GLOMONET. Therefore, the proposed scheme satisfies the security requirements for roaming service and is suitable for practical mobile environments.

Author Contributions: Conceptualization, S.Y.; Formal analysis, J.L., and Y.P. (YoHan Park); Software, S.Y., and J.L.; Supervision, Y.P. (YoungHo Park); Validation, S.L., B.C., Y.P., (YoHan Park) and Y.P. (YoungHo Park); Writing-original draft, S.Y.; Writing-review and editing, S.L., B.C., Y.P., (YoHan Park) and Y.P. (YoungHo Park). All authors have read and agreed to the published version of the manuscript.

Funding: This research was supported by the Institute for Information \& communications Technology Promotion (IITP) grant funded by the Korea government(MIST) (No.2018-0-00312, Developing technologies to predict, detect, respond, and automatically diagnose security threats to automotive Ethernet-based vehicle).

Conflicts of Interest: The authors declare no conflict of interest.

\section{References}

1. Gope, P.; Hwang, T. An efficient mutual authentication and key agreement scheme preserving strong anonymity of the mobile user in global mobility networks. J. Netw. Comput. Appl. 2016, 62, 1-8. [CrossRef]

2. Li, X.; Niu, J.; Kumari, S.; Wu, F.; Choo, K.K.R. A robust biometrics based three-factor authentication scheme for global mobility networks in smart city. Future Gener. Comput. Syst. 2018, 83, 607-618. [CrossRef]

3. Lu, Y.; Xu, G.; Li, L.; Yang, Y. Robust privacy-preserving mutual authenticated key agreement scheme in roaming service for global mobility networks. IEEE Syst. J. 2019, 13, 1454-1465. [CrossRef]

4. Lee, T.F. User authentication scheme with anonymity, unlinkability and untrackability for global mobility networks. Secur. Commun. Netw. 2013, 6, 1404-1413. [CrossRef]

5. Lee, C.C.; Lai, Y.M.; Chen, C.T.; Chen, S.D. Advanced secure anonymous authentication scheme for roaming service in global mobility networks. Wirel. Pers. Commun. 2017, 94, 1281-1296. [CrossRef]

6. He, D.; Ma, M.; Zhang, Y.; Chen, C.; Bu, J. A strong user authentication scheme with smart cards for wireless communications. Comput. Commun. 2011, 34, 367-374. [CrossRef]

7. Jiang, Q.; Ma, J.; Li, G.; Yang, L. An enhanced authentication scheme with privacy preservation for roaming service in global mobility networks. Wirel. Pers. Commun. 2013, 68, 1477-1491. [CrossRef]

8. Kuo, W.C.; Wei, H.J.; Cheng, J.C. An efficient and secure anonymous mobility network authentication scheme. J. Inf. Secur. Appl. 2014, 19, 18-24. [CrossRef]

9. Park, K.S.; Park, Y.H.; Park, Y.H.; Reddy, A.G.; Das, A.K. Provably secure and efficient authentication protocol for roaming service in global mobility networks. IEEE Access 2017, 5, 25110-25125. [CrossRef]

10. Zhu, J.; Ma, J. A new authentication scheme with anonymity for wireless environments. IEEE Trans. Consum. Electron. 2004, 50, 231-235.

11. Lee, C.C.; Hwang, M.S.; Liao, I.E. Security enhancement on a new authentication scheme with anonymity for wireless environments. IEEE Transs. Ind. Electron. 2006, 53, 1683-1687. [CrossRef]

12. Wu, C.C.; Lee, W.B.; Tsaur, W.J. A secure authentication scheme with anonymity for wireless communications. IEEE Commun. Lett. 2008, 12, 722-723.

13. Li, C.T.; Lee, C.C. A novel user authentication and privacy preserving scheme with smart cards for wireless communications. Math. Comput. Model. 2012, 55, 35-44. [CrossRef]

14. Das, A.K. A secure and effective user authentication and privacy preserving protocol with smart cards for wireless communications. Netw. Sci. 2013, 2, 12-27. [CrossRef]

15. Karuppiah, M.; Saravanan, R. A Secure Authentication Scheme with User Anonymity for Roaming Service in Global Mobility Networks. Wirel. Pers. Commun. 2015, 84, 2055-2078. [CrossRef]

16. Madhusudhan, R.; Shashidhara. A secure and lightweight authentication scheme for roaming service in global mobile networks. J. Inf. Secur. Appl. 2018, 38, 96-110. [CrossRef]

17. Dolev, D.; Yao, A. On the security of public key protocols. IEEE Trans. Inf. Theory 1983, 29, 198-208. [CrossRef]

18. Mohit, P.; Amin, R.; Karati, A.; Biswas, G.P.; Khan, M.K. A standard mutual authentication protocol for cloud computing based health care system. J. Med. Syst. 2017, 41, 50. [CrossRef]

19. Amin, R.; Islam, S.K.H.; Biswas, G.P.; Khan, M.K.; Kumar, N. A robust and anonymous patient monitoring system using wireless medical sensor networks. Future Gener. Comput. Syst. 2018, 80, 483-495. [CrossRef]

20. Kocher, P.; Jaffe, J.; Jun, B. Differential power analysis. In Advances in Cryptology-CRYPTO; Lecture Notes in Computer Science; Springer: Santa Barbara, CA, USA, 1999; pp. 388-397. 
21. Yu, S.J.; Lee, J.Y.; Lee, K.K.; Park, K.S.; Park, Y.H. Secure authentication protocol for wireless sensor networks in vehicular communications. Sensors 2018, 18, 3191. [CrossRef]

22. Dodis, Y.; Reyzin, L.; Smith, A. Fuzzy extractors: How to generate strong keys from biometrics and other noisy data. In International Conference on the Theory and Applications of Cryptographic Techniques; Springer: Interlaken, Switzerland, 2004; pp. 523-540.

23. Odelu, V.; Das, A.K.; Goswami, A. An efficient biometric-based privacy-preserving three-party authentication with key agreement protocol using smart cards. Secur. Commun. Netw. 2015, 8, 4136-4156. [CrossRef]

24. Park, Y.H.; Park, Y.H. Three-factor user authentication and key agreement using elliptic curve cryptosystem in wireless sensor networks. Sensors 2016, 16, 2123. [CrossRef] [PubMed]

25. Burrows, M.; Abadi, M.; Needham, R. A logic of authentication. ACM Trans. Comput. Syst. 1990, 8, 18-36. [CrossRef]

26. Abdalla, M.; Fouque, P.A.; Pointcheval, D. Password based authenticated key exchange in the three-party setting. In Public Key Cryptography; Springer: Les Diablerets , Switzerland, 2005; pp. 65-84.

27. Wang, D.; Cheng, H.; Wang, P.; Huang, X.; Jian, G. Zipf's law in passwords. IEEE Trans. Inf. Forensics Secur. 2017, 12, 2776-2791. [CrossRef]

28. Yu, S.J.; Park, K.S.; Lee, J.Y.; Park, Y.H.; Park, Y.H.; Lee, S.W.; Chung, B.H. Privacy-preserving lightweight authentication protocol for demand response management in smart grid environment. Appl. Sci. 2020, 10, 1758. [CrossRef]

29. Park, K.S.; Park, Y.H.; Park, Y.H.; Das, A.K. 2PAKEP: Provably Secure and Efficient Two-Party Authenticated Key Exchange Protocol for Mobile Environment. IEEE Access 2018, 6, 30225-30241. [CrossRef]

30. AVISPA. Automated Validation of Internet Security Protocols and Applications. Available online: http: //www.avispa-project.org/ (accessed on 8 February 2020).

31. SPAN: A Security Protocol Animator for AVISPA. Available online: http://www.avispa-project.org/ (accessed on 8 February 2020).

32. Kumar, V.; Jangirala, S.; Ahmad, M. An efficient mutual authentication framework for healthcare system in cloud computing. J. Med. Syst. 2018, 42, 142. [CrossRef]

33. Chandrakar, P.; Om, H. A secure and robust anonymous three-factor remote user authentication scheme for multi-server environment using ECC. Comput. Commun. 2017, 110, 26-34. [CrossRef]

34. Lee, H.J.; Lee, D.H.; Moon, J.H.; Jung, J.W.; Kang, D.W.; Kim, H.S.; Won, D.H. An improved anonymous authentication scheme for roaming in ubiquitous networks. PLoS ONE 2018, 13, e0193366. [CrossRef]

(C) 2020 by the authors. Licensee MDPI, Basel, Switzerland. This article is an open access article distributed under the terms and conditions of the Creative Commons Attribution (CC BY) license (http:/ / creativecommons.org/licenses/by/4.0/). 